\title{
On Discerning Intermolecular and Intramolecular Vibrations in Experimental Acene Spectra
}

\author{
Faustine Spillebout, ${ }^{\dagger}$ Didier Bégué, ${ }^{\ddagger}$ Isabelle Baraille, ${ }^{\dagger}$ and John M. Shaw ${ }^{*}{ }^{\dagger}$ \\ ${ }^{\dagger}$ Department of Chemical and Materials Engineering, University of Alberta, Edmonton, Alberta, Canada, T6G 2G6 \\ ${ }^{\ddagger}$ Institut des Sciences Analytiques et de Physico-Chimie pour l'Environnement et les Matériaux, Equipe Chimie Physique, UMR 5254, \\ Université de Pau et des Pays de l'Adour, 64000 Pau, France
}

\section{Supporting Information}

ABSTRACT: The far-infrared spectra of the aromatic hydrocarbons tetracene and pentacene have been calculated using a perturbational-variational method coupled with potential truncation. This shortening of the potential enabled accurate vibration calculations for these large molecules and their respective dimers. Thus, it was possible to identify all IR bands obtained experimentally in the far-IR range by Michaelian et al., as well as to differentiate bands resulting from intermolecular and intramolecular modes of vibration separately, and combined intermolecular + intramolecular vibration modes. Far-IR spectra for smaller acene family members, naphthalene and anthracene, were also computed, and trends in intermolecular vibrations, for the acene family as a whole, were identified. The results obtained illustrate the quality and the detail of the insights realized by interrogating experimental spectra using high-precision, unscaled quantum mechanics computational approaches, and provide a benchmark for future work targeting identification of dominant molecular motifs and intermolecular association phenomena arising in ill-defined hydrocarbons including asphaltenes based on IR and Raman spectral decomposition.

\section{INTRODUCTION}

The identification of compounds, compound classes, and principal submolecular motifs present and molecular association phenomena arising in petroleum fractions has been the subject of intense research for many years. ${ }^{1-13}$ However, fluid characterization remains a much debated and critically important topic. Detailed information on the chemical composition and association behavior, particularly of petroleum fractions comprising larger molecules, is needed to predict thermophysical properties, to develop and optimize techniques for hydrocarbon production and refining, and thus to provide opportunities for considerable capital and operating cost reductions and energy efficiency. ${ }^{14,15}$

Vibrational spectroscopy, a widely used experimental technique for the identification and characterization of molecules in complex chemical environments, has been applied successfully in fields as diverse as biochemistry, ${ }^{16-18}$ agribusiness, ${ }^{19-21}$ interstellar chemistry, ${ }^{22,23}$ as well as the chemistry of materials. ${ }^{2-26}$ In these successful applications, knowledge of the molecules constituting the medium of interest is available. For complex and ill-defined mixtures such as petroleum, a combination of experimental measurement and predictive modeling is required. With the joint progress of modeling techniques ${ }^{27-43}$ and computer technology, quantum mechanical calculations have become an essential and powerful contributor for the identification and discrimination of diverse molecular mixtures. ${ }^{44-47}$ Available mathematical techniques have been tested widely and implemented to serve experimentalists. For example, a recent computational study ${ }^{48}$ focused on petroleum sector applications showed that aromatic and naphthenic motifs, present in large molecules, could in principle be discriminated on the basis of the joint decomposition of Raman and IR spectra using a library of small aromatic and naphthenic compounds comprising the motifs, whereas proton and ${ }^{13} \mathrm{C}-\mathrm{NMR}$ provided little information at this length scale.

The work presented here falls within the framework of these mathematical developments to serve identification of complex chemical systems, where the vibrational Schrödinger equation is solved using a double electrical and mechanical anharmonic approximation. Simpler vibration mode calculations, at the harmonic level, are commonly used to predict vibration modes of molecules. ${ }^{49-51}$ However, the resulting error must then be "corrected" using "adapted" multiplicative correction factors that depend on the level of theory employed to solve the Schrödinger equation. These approximate calculations do not allow assignment of modes other than fundamentals, because coupling is not taken into account.

The strategy in the present work is to perform high-precision quantum mechanical calculations based on anharmonic electrical and mechanical approximations to compute spectra for individual molecules and dimers. With this approach, unscaled computed outcomes including harmonics, overtones, combinations, intermolecular vibrations, and other details of spectra can be compared directly with experimental data. The detailed identification of bands in experimental vibration spectra for crude oils and oil fractions may eventually permit the identification of the presence of members of a family of molecules, and the determination of the significance of specific types of molecular interaction arising in these fluids through spectral decomposition. Potential impacts include improved speciation, and hence fluid characterization, and improved

Received: January 7, 2014

Revised: $\quad$ March 27, 2014 
understanding of association phenomena, such as $\pi-\pi$ interactions, and hence transport property prediction.

Acenes, a family of linear polynuclear aromatic compounds possessing only $\pi-\pi$ interactions, for which high precision experimental data are available up to pentacene, ${ }^{52}$ comprise a benchmark example that illustrates both the potential and the computational challenges found in this line of inquiry. In this respect, various calculation hypotheses used for these large molecules are presented, together with the impact of these calculation hypotheses on the quality vis-à-vis experimental data and the computational cost of vibrational spectra.

Spectral assignments, determined on the basis of both wavenumber and relative intensity values, are made primarily using computed spectra for individual molecules, and dimers. In addition, on the basis of experimental observation assessing the presence of deformed molecules in tetracene and pentacene crystals, the impact on vibrational spectra of these deformations is evaluated. For some cases, assignment ambiguity is only resolved by tracing trends for vibration modes for the acene family as a whole. In the exposition that follows, each of these topics is tackled sequentially. Calculations with tetracene and pentacene monomers (individual molecules) are treated first. Then, calculations for dimers and the acene family collectively are treated, and the results synthesized.

\section{- CALCULATION OF VIBRATIONAL SPECTRA OF TETRACENE AND PENTACENE MONOMERS: COMPARISON WITH EXPERIMENTAL DATA}

a. Experimental Data. Experimental solid-state infrared spectra of tetracene and pentacene ${ }^{52}$ were acquired at room pressure and temperature. The measurements were performed on as-received samples. Thus, the crystallinity as well as the possible presence of defects in these solids are unknown. For tetracene, two crystal structures have been detected by X-ray diffraction experiments. ${ }^{53}$ At room temperature and pressure, tetracene is triclinic ${ }^{54}$ with space group $P \overline{1}$. The unit cell contains two independent molecules at the $(0,0,0)$ and $(1 / 2$, $1 / 2,0)$ inversion sites. Pentacene possesses two polymorphs. ${ }^{55,56}$ One comprises a triclinic layered structure with a herringbone arrangement in the layers, with two equivalent molecules per unit cell. ${ }^{57}$ The other, commonly found at room temperature, adopts a $14.1 \AA d(001)$-spacing morphology with an inversion center on both molecules in the unit cell. ${ }^{58-62}$

b. Computational Details: Construction of the Potential Energy Surface. The theoretical process generally developed to resolve the vibrational Schrödinger equation requires two restricted steps: the construction of the potential energy surface (PES) and the resolution of the vibrational equation in order to obtain the harmonic and anharmonic energy levels and consequently the fundamental, overtone, and harmonic wavenumbers characterizing the vibrational spectra. Calculations of the intensities complete the analysis of the spectra.

The potential energy surface is normally constructed by expressing the potential function as a Taylor series expansion using curvilinear displacement coordinates. This series is often truncated at the fourth order. Quadratic, cubic, and quartic force constants are generally obtained by fitting the electronic energy data calculated by $a b$ initio methods for various nuclear configurations close to the optimized geometry, or by a finite difference procedure for first or second derivatives of the electronic energy with respect to the nuclear coordinates. ${ }^{63}$
The Becke three-parameter hybrid functional (B3LYP), a combination of the Becke three-parameter exchange functional (B3) ${ }^{64}$ that includes a mixture of Hartree-Fock exchange with the density functional theory (DFT) exchange-correlation and the LYP correlation functional, ${ }^{65}$ has proven to be a reasonable choice for predicting geometries of aromatic molecules. ${ }^{66,67}$ Stephens et al. ${ }^{68}$ showed that the B3LYP force field yields infrared spectra in very good agreement with experimental data. It has become a standard method for studying vibrational spectra of organic molecules (in the far- and mid-infrared) in the gas phase. ${ }^{49,69}$ Although the B3LYP method coupled with a sufficiently large basis set is a good candidate to study vibrational spectra of polynuclear aromatic molecules, ${ }^{52}$ this standard DFT calculation approach fails to describe longdistance interactions. Consequently, it does not reproduce spectra of interacting molecules ${ }^{64,68,70,71}$ where the interpretation of experimental vibration spectra requires consideration of both isolated and interacting molecules.

In parallel to the development to $a b$ initio methods, some progress has been made on implementing the London dispersion force effect into DFT methods, permitting calculations with larger individual molecules and binary pairs. Although the $\operatorname{CCSD}(\mathrm{T})$ method in combination with a sufficiently flexible basis set is the most accurate method for reproducing rotation-vibration spectra and to describe intermolecular complexes between aromatic hydrocarbons, ${ }^{72}$ such calculations are impractical for the description of large molecules. ${ }^{73}$ The MP2 method, ${ }^{74}$ also able to handle dispersive interactions such as $\pi-\pi$ stacking, ${ }^{75,76}$ is a computationally intensive and inadequate method for large molecules too. ${ }^{77}$ Chai et al. ${ }^{78}$ developed a functional based on optimized longrange corrected hybrid density functionals, ${ }^{79}$ which employs $100 \%$ Hartree-Fock (HF) exchange for long-range electronelectron interactions, $\omega \mathrm{B} 97 \mathrm{X}$, to which they added an empirical dispersive interaction correction. ${ }^{77}$ This method was tested by Salzner et al. ${ }^{80}$ for $\pi$-conjugated oligomers, and it overcame the difficulties encountered with standard DFT functionals when dealing with interacting $\pi$-systems.

In this work, extended basis sets consisting of atomic orbitals expressed as fixed linear combinations of Gaussian functions are employed for the calculations. A split valence 6-311G and 6$311 \mathrm{G}(\mathrm{p}, \mathrm{d})^{81,82}$ was needed to provide correct calculation outcomes for naphthalene monomer. ${ }^{50}$ Validation tests for the $\omega \mathrm{B} 97 \mathrm{X}-\mathrm{D} / 6-311 \mathrm{G}$ calculation method were also performed. The accuracy of DFT calculations in predicting molecular geometries and vibrational frequencies depends on the density functional employed. Thus, geometrical optimizations were performed for isolated tetracene, using the $\omega \mathrm{B} 97 \mathrm{X}-\mathrm{D}$ functional as well as the more conventional B3LYP method. The computational results are benchmarked using experimental data obtained by Campbell et al. ${ }^{54}$

c. Geometrical Parameters of Isolated Tetracene Molecule Optimized Using B3LYP and $\omega$ B97X-D Methods Associated with the 6-311G and 6-311G** Basis Sets. Geometry optimizations were performed using two different DFT methods (B3LYP and $\omega$ B97X-D) implemented in Gaussian 09 software ${ }^{83}$ and two basis sets (6-311G and 6$\left.311 \mathrm{G}^{* *}\right)$. While Saeki et al. ${ }^{50}$ reported a change of symmetry for the naphthalene molecule, from $D_{2 h}$ to $C_{2 h}$ (based on MP2/ 6-311G calculations), optimized geometries for the tetracene molecule, which also possesses $D_{2 h}$ symmetry, remained planar with $D_{2 h}$ symmetry in all cases. Calculated bond lengths based on different levels of theory are listed in Table 1 along with 
Table 1. Method and Basis-Set Dependence of C-C Bond Length $(\AA)^{a}$ for Tetracene Monomer and Mean Deviation from Experimental Data ${ }^{b}$

\begin{tabular}{lllllllll} 
& \multicolumn{1}{c}{$\mathrm{C} 1-\mathrm{C} 2$} & $\mathrm{C} 2-\mathrm{C} 3$ & $\mathrm{C} 3-\mathrm{C} 4$ & $\mathrm{C} 4-\mathrm{C} 5$ & $\mathrm{C} 4-\mathrm{C} 6$ & $\mathrm{C} 6-\mathrm{C} 7$ & C7-C8 & mean deviation \\
experimental data $^{a}$ & 1.46 & 1.38 & 1.42 & 1.42 & 1.39 & 1.40 & 1.46 \\
B3LYP/6-311G & $1.43(-0.03)$ & $1.36(-0.02)$ & $1.43(+0.01)$ & $1.43(+0.01)$ & $1.40(+0.01)$ & $1.42(+0.02)$ & $1.43(-0.03)$ & 0.02 \\
B3LYP/6-311G** & $1.43(-0.03)$ & $1.36(-0.02)$ & $1.43(+0.01)$ & $1.43(+0.01)$ & $1.40(+0.01)$ & $1.42(+0.02)$ & $1.43(-0.03)$ & 0.03 \\
$\omega$ B97X-D/6-311G & $1.43(-0.03)$ & $1.37(-0.02)$ & $1.44(+0.02)$ & $1.45(+0.03)$ & $1.39(=)$ & $1.41(+0.01)$ & $1.45(-0.01)$ & 0.02 \\
$\omega$ B97X-D/6-311G** & $1.43(-0.03)$ & $1.37(-0.02)$ & $1.44(+0.02)$ & $1.45(+0.02)$ & $1.39(=)$ & $1.41(+0.01)$ & $1.45(-0.01)$ & 0.02
\end{tabular}

${ }^{a_{T}}$ The values in parentheses indicate the difference between the calculated and experimental bond lengths. ${ }^{b}$ Experimental bond lengths are obtained from ref 54 .

their mean deviations from experimental measurements (see also Figure S1 in the Supporting Information). For these calculations, irrespective of the level of theory or the basis set employed, the agreement between computed bond lengths and experimental values is very good. The mean deviation from experimental values is less than $0.03 \AA$. These results show clearly that the choice of calculation method and basis set has a minor influence on the geometry of tetracene. Results obtained with pentacene monomer are comparable. This basis-set insensitivity for DFT approaches has been illustrated previously. ${ }^{84}$ It permits the use of smaller basis sets and provides an additional argument for the use of DFT over $a b$ initio methods, since larger molecules can be treated. The $\omega$ B97X-D method, which contains a London-force dispersive term and is reliable for acene molecules, was selected for this study, and it is used in combination with the 6-311G basis set.

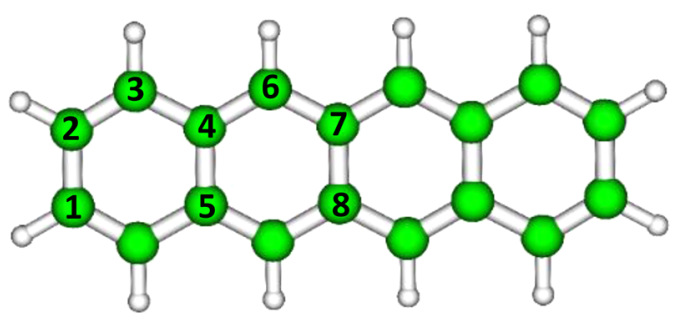

Figure 1. Representation of a tetracene molecule.

d. Truncation of the Potential Energy Surface.

Vibrational spectroscopy modeling outcomes, whether at the harmonic or anharmonic level, are directly dependent on the quality of the potential energy surface (PES). The PES is generally expressed as a polynomial determined by adjustment of a set of geometric structures of a molecule. Consequently, this type of calculation depends on the quality of the electronic calculations of the wave functions as well.

While it is now common to determine the PES accurately for small molecules ( $3-5$ atoms) ${ }^{85}$ this becomes almost infeasible when solving anharmonic approximations for systems of larger size where powerful computers are required to implement rigorous methods for solving the Schrödinger equation. Truncation of the analytical shape of the PES (degree of the potential) is unavoidable. For example, a tetracene molecule contains 30 atoms, and therefore has $3 N-6=84$ normal modes apart from the translational and rotational modes. 84 modes with an order 4 necessitate the calculation for 22171 force constants. Thus, truncation of the PES constitutes the first part of this study. Four levels of approximation were tested. The fundamental bands obtained in these cases, using secondorder perturbation theory (PT2), are compared to the ones calculated with the complete PES. The conclusions of these tests are summarized in Table 2 and elaborated here:

(i) It is possible to truncate the PES in terms of only significant coupling. For this, it suffices to identify the constant of cubic and quartic force of very low intensity.

(ii) We have realized calculations by successively suppressing force constants smaller than $2,5,8$, and $10 \mathrm{~cm}^{-1}$. This study showed that the discrepancy regarding the calculated frequencies of fundamental modes is not higher than 2.8 and $3.3 \mathrm{~cm}^{-1}$ for the combination modes. These disparities are small enough to justify use of this approximation. Moreover, this allows setting $7,33,48$, and $55 \%$ of the forces to zero when

Table 2. Force Constant Truncation and Separation of Low and High Mode Influences on the Values of Wavenumbers of Tetracene Monomer Based on a Force Field Extracted from Calculations at the $\omega$ B97X-D/6-311G Level of Theory

polynomial degree
force constants $(f)$ considered
number of modes
mode (symmetry)
$\nu_{1}\left(\mathrm{~B}_{3 \mathrm{u}}\right)$
$\nu_{2}\left(\mathrm{~A}_{\mathrm{u}}\right)$
$\nu_{3}\left(\mathrm{~B}_{1 \mathrm{~g}}\right)$
$\nu_{4}\left(\mathrm{~B}_{1 \mathrm{u}}\right)$
$\nu_{5}\left(\mathrm{~B}_{2 \mathrm{~g}}\right)$
$\nu_{6}\left(\mathrm{~B}_{3 \mathrm{u}}\right)$
$\nu_{7}\left(\mathrm{~B}_{3 \mathrm{~g}}\right)$
$\nu_{8}\left(\mathrm{~A}_{\mathrm{g}}\right)$
$\nu_{9}\left(\mathrm{~A}_{\mathrm{u}}\right)$
$\nu_{10}\left(\mathrm{~B}_{1 \mathrm{~g}}\right)$

$\begin{array}{lll} & 2 & \begin{array}{l}4^{c} \\ \text { all } f \text { con } \\ \end{array} \\ & & 84 \\ \text { exp. values }^{a} & & \\ & & \\ & 57 & 48 \\ & 93 & 90 \\ 166^{b} & 154 & 150 \\ & 168 & 169 \\ 271^{b} & 196 & 194 \\ & 276 & 272 \\ 322^{b} & 316 & 317 \\ & 324 & 322 \\ & 327 & 325 \\ & 389 & 382\end{array}$

$4^{c}$
$f>5 \mathrm{~cm}$
84
53
96
154
160
200
277
305
323
332
386

$4^{c}$
$f>5 \mathrm{~cm}$
20
62
107
161
169
204
284
317
322
330
395

$4^{c}$
$f>5 \mathrm{~cm}$
10
60
103
160
169
202
282
317
320
328
392

$6^{c}$
all $f$ considered
10
69
107
164
171
203
284
319
322
331
394

${ }^{a}$ Experimental values of wavenumbers are obtained from ref $52 .{ }^{b}$ Experimental uncertainty equal to $\pm 2 \mathrm{~cm}^{-1}$. ${ }^{c}$ Wavenumbers calculated using second-order perturbation theory (PT2). 
suppressing constants smaller than $2,5,8$, and $10 \mathrm{~cm}^{-1}$, respectively, thus saving computational cost and enabling variational calculations for larger systems. Note, however, that this restriction is potentially acceptable only without any Fermi or Darling-Dennison resonance, often characterized totally or partially using these finest couplings. Regarding the time saved when suppressing the forces lower than $10 \mathrm{~cm}^{-1}$ while the wavenumber values of fundamental modes remain the same, it would be tempting to truncate the PES this way, but this would represent a serious risk of losing information regarding combination bands. Indeed, our tests show that the upper limit of the truncation to maintain all the information on combination bands consists of considering all the forces higher than $5 \mathrm{~cm}^{-1}$. Removal of these forces would disregard combinations whose weight is less important, resulting in the loss of details that provide a complete interpretation of infrared spectra. Thus, for the following tests of truncature and for calculations presented in this study, we will consider a PES calculated with force constants higher than $5 \mathrm{~cm}^{-1}$.

(iii) The second truncation tested in this work consists of separating modes of high and low wavenumber. We assumed that the low-wavenumber modes (mainly torsion modes) were weakly coupled with bending and scissoring modes occurring at higher wavenumber. This hypothesis has been tested and validated on the basis of calculations on the tetracene molecule (Table 2); indeed, each of the first 15 modes calculated using the PES truncated to torsional and libration modes are in perfect adequacy with values calculated a posteriori using complete PES. Figure S2 in the Supporting Information shows that the effect of suppressing the higher modes has a minor incidence on the values of the fundamental modes calculated at the vibrational MP2 level. However, suppressing modes higher than $500 \mathrm{~cm}^{-1}$ was even beneficial when considering combination bands. Indeed, calculations can cause the modes of higher frequencies, with large amplitude, to interfere artificially on these "soft modes" localized in the range $[0$, $400] \mathrm{cm}^{-1}$, giving rise to nonphysical frequencies. Another consequence of the artificial interactions between these modes is that combination modes of low wavenumber are embedded in the parasitic values of frequencies, and then result in loss of information.

(iv) We have also tested the association of a complete grid of points of the surface at the fourth order, with a fit at the sixth order on the diagonal, in order to refine coupling between closely coupled modes. Because this refining is demanding in terms of computing time, we applied it on the 10 first modes only, supposing that the modes of higher wavenumber were correctly described with a polynome of fourth order. The results, listed in Table 2, show that this refining does not bring any improvement for describing the vibrational spectrum of tetracene.

The last approximation used in this work consists of applying a second adjustment of the PES; this adjustment would aim to eliminate the weight of vibrators we excluded in point $i$, to refine the fit of remaining force constants so that they reflect, in fine, only the couplings between remaining modes.

If this had proved to be an acceptable approximation, we could have extended this methodology to the study of specific molecule vibrators of undifferentiated dimension, by calculating a limited number of specific points to sought vibrators.

In accordance with what Bowman et al. ${ }^{86}$ and Begue et al. ${ }^{87}$ showed on the study of water clusters, our calculations show that only the separation between libration modes with all other active modes is possible.

To conclude, we have shown that partial truncation of PES could be effective for our study. This truncation consists of extracting from a global fit the force constants of higher order that allow the description of the problem beyond the harmonic approximation. These constants can then be directly used to solve Schrödinger's vibrational equation.

This methodology was used in the remainder of our work for the characterization and assignment of the spectra of tetracene and pentacene molecules.

e. Resolution of the Vibrational Schrödinger Equation (Noncore Bands with Intensity). The presence of overtones and combination bands in infrared spectra is a manifestation of the breakdown of the double-harmonic approximation, usually implemented in electronic structure codes. The calculation of intensities is essential in order to determine which nonfundamental transitions are active and to assign all experimental bands. Since both mechanical (anharmonicity of the potential) and electrical (nonlinear dependence of the dipole moment on the normal coordinates) anharmonicities are expected to give nonzero intensities to nonfundamental transitions, these two effects must be considered in the treatment of transition energies and related vibrational wave functions. The wavenumber calculations in the mechanical anharmonic approximation were carried out using a variational method developed by Bégué et al. ${ }^{85}$ Using this method, implemented in the P Anhar.v2.0 program, ${ }^{88}$ it was possible to compute all the vibrational frequencies (fundamental, combination bands, and overtones) that contribute to the mid- and near-infrared spectrum of tetracene and pentacene. In addition, the activity of each mode was also calculated in the electrical anharmonic approximation using a method developed by Baraille et al., ${ }^{87,89}$ which is also available in the same software.

The experimental spectra of tetracene and pentacene, extracted from the work of Michaelian et al., ${ }^{52}$ are shown in Figure 2. The corresponding calculated infrared-active transitions - both fundamental and combination-overlay the data and are listed in Tables 3 and 4 where they are further compared with available experimental and theoretical values. A

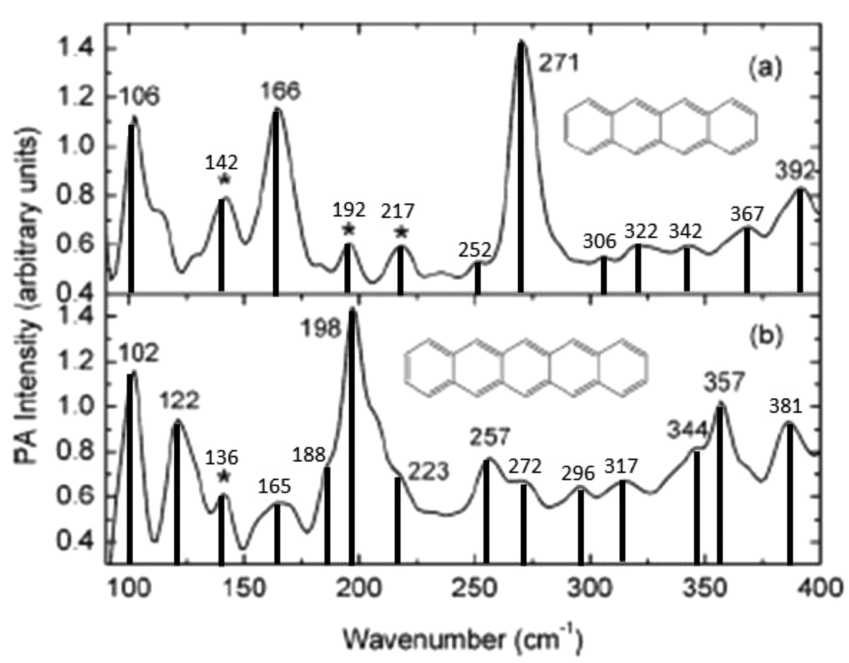

Figure 2. Far-infrared PA spectra of (a) tetracene and (b) pentacene. Spectra were acquired using phase modulation at a frequency of $5 \mathrm{~Hz}$ and an amplitude of $15 \lambda(\lambda=0.6328 \mu \mathrm{m})$, extracted from Michaelian et al. ${ }^{52}$ 
Table 3. Experimental and Calculated Wavenumbers $\left(\mathrm{cm}^{-1}\right)$ for Fundamental and Combination Vibrational Modes of Tetracene $^{d}$

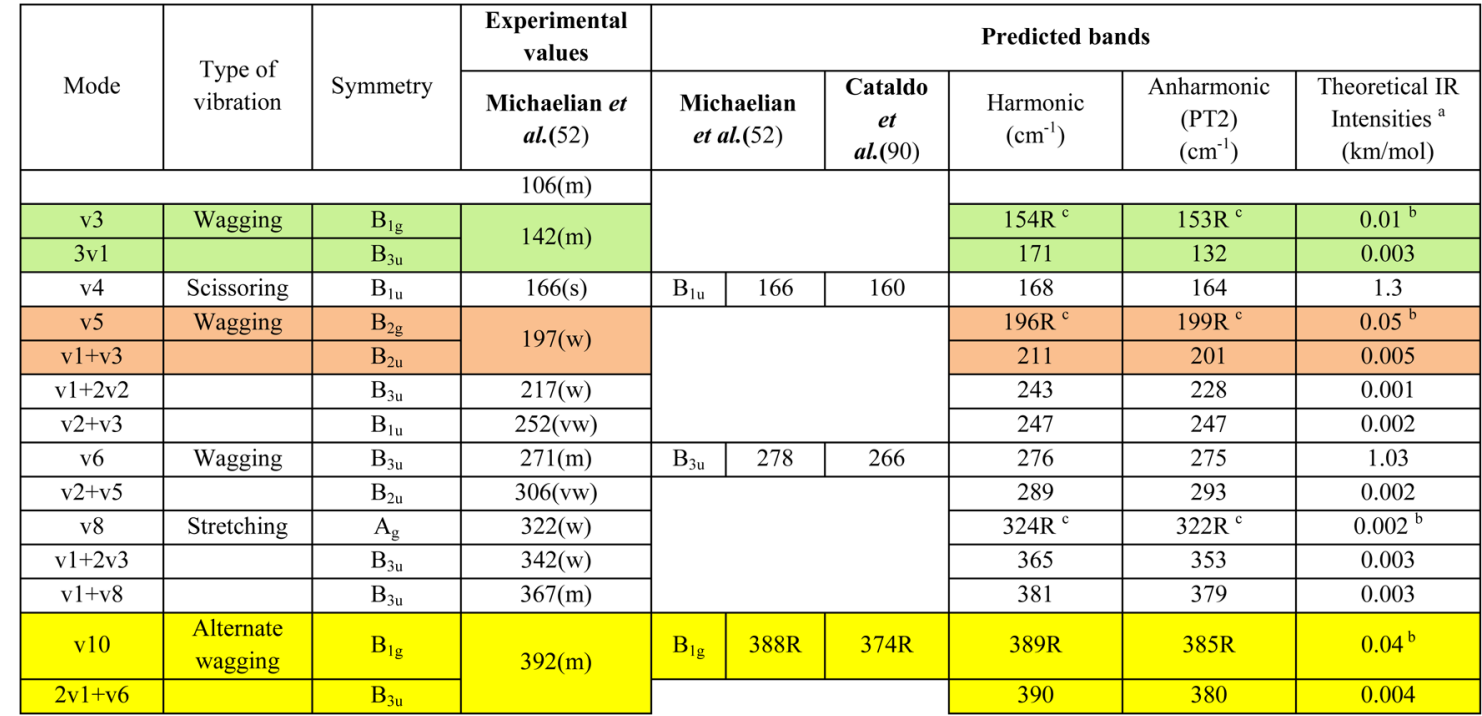

${ }^{a}$ Theoretical intensities obtained using perturbational-variational calculations, with truncated potential on the 20 first modes. ${ }^{b}$ Theoretical intensities obtained for the distorted molecule, at the harmonic level. ${ }^{c}$ The letter $\mathrm{R}$ indicates Raman-active modes. ${ }^{d}$ Colored lines indicate cases with doublehypotheses.

Table 4. Experimental and Calculated Wavenumbers $\left(\mathrm{cm}^{-1}\right)$ for Fundamental and Combination Vibrational Modes for Pentacene $^{d}$

\begin{tabular}{|c|c|c|c|c|c|c|c|c|c|}
\hline \multirow[b]{2}{*}{ Mode } & \multirow{2}{*}{$\begin{array}{l}\text { Type of } \\
\text { vibration }\end{array}$} & \multirow[b]{2}{*}{ Symmetry } & Experimental & \multicolumn{6}{|c|}{ Predicted bands } \\
\hline & & & $\begin{array}{l}\text { Michaelian } \\
\text { et al. (52) }\end{array}$ & \multicolumn{2}{|c|}{$\begin{array}{l}\text { Michaelian et al. } \\
\text { (52) }\end{array}$} & $\begin{array}{c}\text { Cataldo } \\
\text { et al. } \\
(90)\end{array}$ & $\begin{array}{l}\text { Harmonic } \\
\left(\mathrm{cm}^{-1}\right)\end{array}$ & $\begin{array}{c}\text { Anharmonic } \\
(\mathrm{PT} 2) \\
\left(\mathrm{cm}^{-1}\right)\end{array}$ & $\begin{array}{c}\text { Theoretical IR } \\
\text { Intensities } \\
(\mathrm{km} / \mathrm{mol})\end{array}$ \\
\hline $\mathrm{v} 3$ & Wagging & $\overline{B_{1 g}}$ & $102 \mathrm{~m}$ & $\mathrm{~B}_{1 \mathrm{~g}}$ & $105 \mathrm{R}^{\mathrm{c}}$ & $100 \mathrm{R}^{\mathrm{c}}$ & $104 R^{c}$ & $97 \mathrm{R}^{\mathrm{c}}$ & $0.003^{b}$ \\
\hline v4 & Scissoring & $\mathrm{B}_{1 \mathrm{u}}$ & $122 \mathrm{~m}$ & $\mathrm{~B}_{\text {lu }}$ & 121 & 117 & 123 & 122 & 1.0 \\
\hline $\mathrm{v} 1+\mathrm{v} 3$ & & $\overline{B_{2 u}}$ & $141 \mathrm{~m}$ & & & & 142 & 144 & 0.004 \\
\hline $\mathrm{v} 2+\mathrm{v} 3$ & & $\mathrm{~B}_{\text {lu }}$ & $165 \mathrm{vw}$ & & & & 178 & 164 & 0.0005 \\
\hline $\mathrm{v} 6$ & Wagging & $\mathrm{B}_{3 \mathrm{u}}$ & $198 \mathrm{~m}$ & $\mathrm{~B}_{3 \mathrm{u}}$ & 198 & 189 & 198 & 194 & 1.4 \\
\hline $\mathrm{v} 3+\mathrm{v} 4$ & & $\mathrm{Au}$ & \multirow{2}{*}{$223 \mathrm{vw}$} & $\mathrm{A}_{\mathrm{u}}$ & & & 224 & & 0 \\
\hline $\mathrm{v} 2+\mathrm{v} 5$ & & $\overline{B_{2 u}}$ & & & & & 226 & 222 & 0.002 \\
\hline v9 & $\begin{array}{l}\text { Symmetric } \\
\text { stretching }\end{array}$ & $A_{g}$ & \multirow[t]{2}{*}{$257 \mathrm{w}$} & $\mathrm{A}_{\mathrm{g}}$ & $266 R^{c}$ & $258 \mathrm{R}^{\mathrm{c}}$ & $269 R^{c}$ & $265 \mathrm{R}^{\mathrm{c}}$ & $0.03^{\mathrm{b}}$ \\
\hline $\mathrm{v} 1+2 \mathrm{v} 3$ & & $\overline{B_{3 u}}$ & & & & & 247 & 251 & 0.0006 \\
\hline $2 v 1+v 6$ & & $\mathrm{~B}_{3 \mathrm{u}}$ & $272 \mathrm{vw}$ & & & & 274 & 274 & 0.0007 \\
\hline $\mathrm{v} 3+\mathrm{v} 6$ & & $\mathrm{~B}_{2 \mathrm{u}}$ & \multirow{2}{*}{$296 \mathrm{w}$} & & & & 302 & 291 & 0.003 \\
\hline $\mathrm{v} 1+\mathrm{v} 9$ & & $\mathrm{~B}_{3 \mathrm{u}}$ & & & & & 307 & 292 & 0.003 \\
\hline $\mathrm{v} 2+\mathrm{v} 7$ & & $\mathrm{~B}_{3 \mathrm{u}}$ & $317 \mathrm{w}$ & & & & 321 & 316 & 0.0009 \\
\hline $\mathrm{v} 3+\mathrm{v} 8$ & & $\overline{\mathrm{B}_{\mathrm{lu}}}$ & $344 \mathrm{vw}$ & & & & 351 & 346 & 0.03 \\
\hline v12 & Scissoring & $\mathrm{B}_{\text {lu }}$ & $357 \mathrm{w}$ & $\mathrm{B}_{\text {lu }}$ & 369 & 357 & 375 & 374 & 0.1 \\
\hline v13 & $\begin{array}{l}\text { Alternate } \\
\text { wagging }\end{array}$ & $\mathrm{B}_{3 \mathrm{u}}$ & $381 \mathrm{~m}$ & & & & 385 & 374 & 0.03 \\
\hline
\end{tabular}

${ }^{a}$ Theoretical intensities obtained using perturbational-variational calculations, with truncated potential on the 20 first modes. ${ }^{b}$ Theoretical intensities obtained for the distorted molecule, at the harmonic level. ${ }^{c}$ The letter $\mathrm{R}$ indicates Raman-active modes. ${ }^{d}$ Colored lines indicate cases with doublehypotheses.

threshold intensity for IR activity of $0.001 \mathrm{~km} / \mathrm{mol}$ was chosen for the calculations. This threshold is justified following a discussion of band assignments for each molecule, and presentation of vibrational bands common for the two molecules.

For tetracene molecules, three bands were identified previously in the range $100-400 \mathrm{~cm}^{-152,91}$ Two bands (166 and $271 \mathrm{~cm}^{-1}$ ) were noted as infrared-active, and one (388 $\mathrm{cm}^{-1}$ ) was assigned as Raman-active (Table 3). By calculating intensities for all bands, including combination bands and overtones, eight additional infrared-active vibrations (seven combinations and one second-harmonic) are identified, and the band at $388 \mathrm{~cm}^{-1}$ previously assigned as Raman-active is identified as an infrared-active band arising from the $\mathrm{B}_{3 \mathrm{u}}$ mode plus an overtone. Three similar cases where a vibrational transition could correspond either to a combination band, an overtone, or a Raman-active band are also shown in rows with colored backgrounds in Table 3. The hypothesis of Raman 
activity of these bands requires further elaboration prior to performing a definitive assignment of these bands, based on their intensities, and where necessary wavenumber trends for bands common to both tetracene and pentacene.

Raman-Transition Activity in Infrared Spectra: Molecular Deformation. Raman transitions can become active in infrared spectra, when a molecule is deformed in the solid state. For example, Homes et al. ${ }^{58}$ showed experimentally that acene molecules in the solid state are not planar and attributed this observation to crystal-packing forces that induce deformation. Further, deformation affects measured lattice-phonon frequencies, permitting discrimination of different solid phases of tetracene $^{53}$ and pentacene. ${ }^{55,92}$ In order to underpin this contention, calculations on the deformed tetracene molecule shown in Figure 3 were performed. In this conformation, the

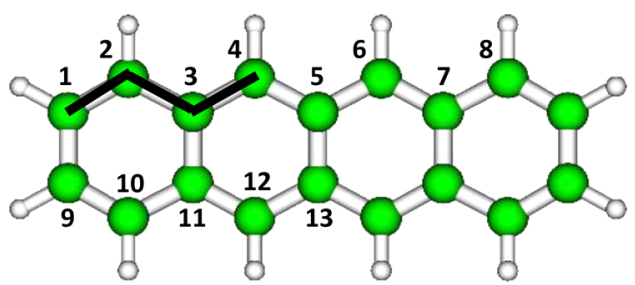

Figure 3. Representation of a tetracene molecule with a deformed dihedral angle $\mathrm{C}_{1}-\mathrm{C}_{2}-\mathrm{C}_{3}-\mathrm{C}_{4}$ shown in black.

molecule does not possess $D_{2 h}$ symmetry, but the molecule does still possess symmetry, because the deformation concerns dihedral angles $\left[\mathrm{C}_{1}-\mathrm{C}_{2}-\mathrm{C}_{3}-\mathrm{C}_{4}\right],\left[\mathrm{C}_{2}-\mathrm{C}_{3}-\mathrm{C}_{4}-\mathrm{C}_{5}\right],\left[\mathrm{C}_{4}-\mathrm{C}_{5}-\right.$ $\left.\mathrm{C}_{6}-\mathrm{C}_{7}\right], \quad\left[\mathrm{C}_{5}-\mathrm{C}_{6}-\mathrm{C}_{7}-\mathrm{C}_{8}\right], \quad\left[\mathrm{C}_{9}-\mathrm{C}_{10}-\mathrm{C}_{11}-\mathrm{C}_{12}\right]$, and $\left[\mathrm{C}_{10}-\right.$ $\left.\mathrm{C}_{11}-\mathrm{C}_{12}-\mathrm{C}_{13}\right]$, equal to $175,178,-178,-178,-178$, and $-178^{\circ}$, respectively, instead of $\pm 180^{\circ}$ in the case of the molecule in the $D_{2 h}$ conformation. The bond lengths are unchanged. Wavenumbers of fundamental modes and associated intensities calculated with the harmonic approximation for deformed tetracene are listed in Table S1 in the Supporting Information. The calculated vibrational spectrum exhibits only positive wavenumbers, which attests that this deformation is located at a minimum of the potential energy surface. The intensities corresponding to the R-denominated bands of the deformed molecule, calculated at the harmonic level, are given in Table 3. For the deformed molecule, modes that would normally be inactive in infrared (Raman-active or silent modes) present significant intensities, ranging from 0.002 to $0.05 \mathrm{~km} / \mathrm{mol}$.

Assignment Based on Band Intensity Value. For tetracene, three transitions for which there is a double-hypothesis (142, 192 , and $392 \mathrm{~cm}^{-1}$ ) can be resolved on the basis of relative intensity values. The band at $142 \mathrm{~cm}^{-1}$ could be attributed to a second harmonic whose intensity would be 3 times lower than the Raman-active one that becomes optically active for a deformed molecule. The band at $192 \mathrm{~cm}^{-1}$ could be a combination of an IR-active mode and a Raman-active mode, whose intensity would be 10 times lower than a Raman-active band calculated at $196 \mathrm{~cm}^{-1}$. The band at $392 \mathrm{~cm}^{-1}$ could either be a combination of an overtone and an infrared-active mode or a Raman-active band 10 times more intense. From the relative intensities, in each case, the hypothesis of a Raman band becoming active is more likely. For other transitions, there is a single hypothesis. The transition, denoted $\mathrm{v}_{8}$, at $322 \mathrm{~cm}^{-1}$, is a Raman-active band, while bands at 252,306 , and $342 \mathrm{~cm}^{-1}$ are combination bands.

The interpretation of results for pentacene, Table 4, is similar. Eleven bands arise from combination vibrations. One, found experimentally at $257 \mathrm{~cm}^{-1}$, was previously assigned to a predicted Raman-active mode. Calculations on a deformed pentacene molecule lead to an IR-active band at a comparable wavenumber and with a comparable intensity. Thus, this one assignment remains uncertain on the basis of these calculations.

Use of Wavenumber Trends to Assign Bands. The trends for normal modes of infrared-active vibrations found for both tetracene and pentacene molecules (see types of vibration in Tables 3 and 4) are shown in Figure 4 where they are also compared with experimental data. By viewing the wavenumbers in this way, wavenumber assignments for the two molecules can be evaluated in parallel. From the similarity of the trends for tetracene and pentacene, as well as the proximity of the

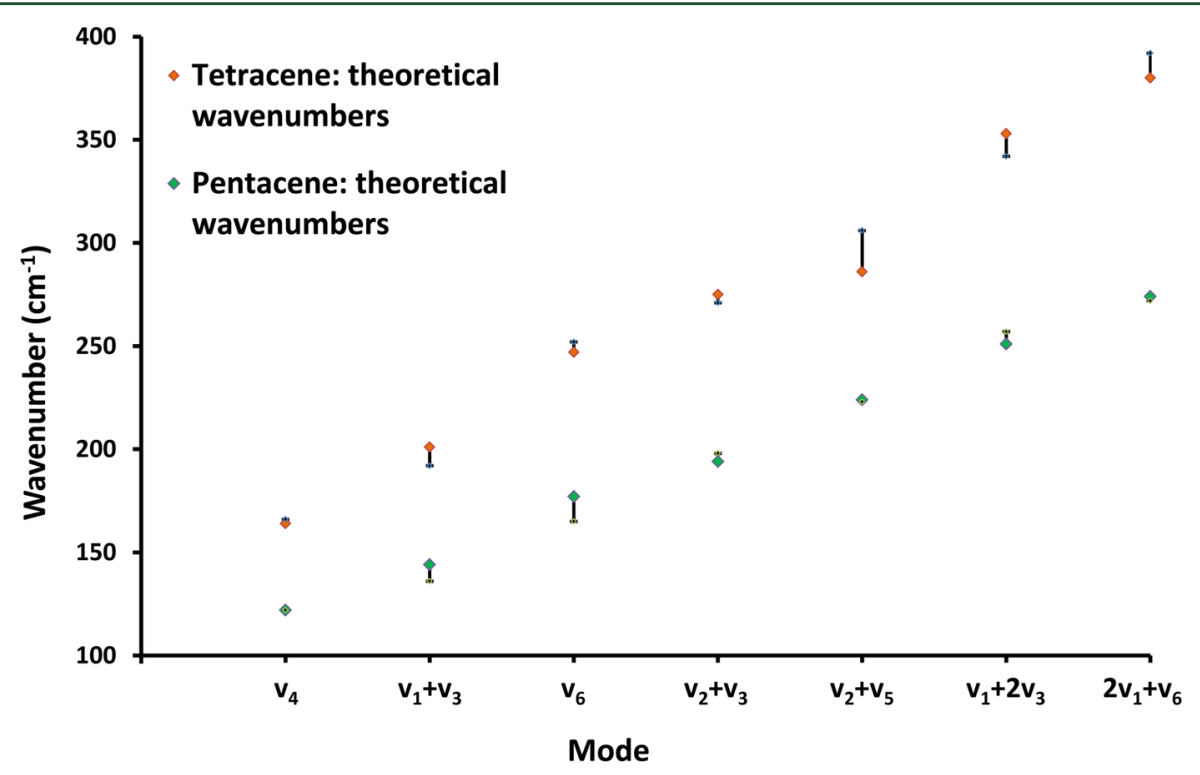

Figure 4. Comparison of theoretical and experimental wavenumbers of tetracene and pentacene for the same normal modes of vibration. The deviations between experimental and theoretical wavenumbers are represented with dark bars. 
respective computed wavenumbers to experimental ones, the assignment choices-combination bands over Raman-active ones-are supported and this outcome is independent of intensity values. Only one band remains unassigned in the experimental spectrum of tetracene, $106 \mathrm{~cm}^{-1}$. Michaelian et al. ${ }^{52}$ suggested that it originated from lattice vibration. The pentacene band attributed to a Raman-active vibration, 102 $\mathrm{cm}^{-1}$, may be of the same type as well. These bands and intermolecular bands more broadly are addressed by performing calculations with tetracene and pentacene dimers.

\section{- CALCULATION OF VIBRATIONAL SPECTRA OF ACENE DIMERS: COMPARISON WITH EXPERIMENTAL DATA}

$\pi$-Stacking is responsible for the cohesive structure of acene crystals. ${ }^{54,60,93-95}$ Dimers constitute a benchmark for describing $\pi$-stacking phenomena. In order to generalize the effect of $\pi$ stacking on intermolecular vibrations for the acene family as a whole, the calculations described here include those for naphthalene and anthracene dimers in addition to the tetracene and pentacene dimers. Inclusion of these smaller dimers also permits calibration of the computational methodology employed.

a. Calculated Geometry and Wavenumbers of Dimers and Comparison with Experimental Data. Far-infrared spectra provide information about intermolecular and inter-ring interactions and about $\pi$-stacking in particular. Hineno et al. ${ }^{96}$ measured spectra of naphthalene and anthracene in the region $190-50 \mathrm{~cm}^{-1}$ at liquid helium temperature and observed that some bands were due to lattice vibrations. In the same way, bands in the experimental vibrational spectra of tetracene and pentacene that cannot be interpreted on the basis of computed wavenumbers for a single molecule are expected to arise from lattice modes. A first step toward understanding $\pi-\pi$ interaction of acene compounds consists of considering the interaction of acene dimers. The choice of the calculation method for describing these electronic interactions is justified, and then, the differentiation of intermolecular and intramolecular vibrations in the acene family is addressed.

Naphthalene Dimer. To assess the performance of the $\omega \mathrm{B} 97 \mathrm{X}-\mathrm{D}$ method for $\pi-\pi$ interaction calculations involving aromatic molecules, the robustness of the $\omega \mathrm{B} 97 \mathrm{X}-\mathrm{D} / 6-311 \mathrm{G}$ method for naphthalene dimers, for which a broad literature exists, ${ }^{50,97-100}$ is discussed in light of results based on reliable data and accurate computational results. For example, Gonzalez et al. $^{97}$ and Tsuzuki et al. ${ }^{98}$ calculated ground-state structures and binding energies of van der Waals dimers of naphthalene using an aromatic intermolecular interaction model, at the MP2 and $\operatorname{CCSD}(\mathrm{T})$ levels of theory, respectively, using Pople's basis sets of various sizes. Their calculations yield two low energy dimers of similar energy, $D_{2 d}$ (crossed) and $C_{2 h}$ (paralleldisplaced), and two $C_{2 v}$ T-shaped structures. Walsh et al., ${ }^{99}$ using calculations at the MP2/6-31*(0.25) level, found eight stationary points for naphthalene dimers: the four reported previously and four others of lower symmetry. Following these studies, Saeki et al. ${ }^{50}$ performed vibrational analysis of naphthalene dimers at the MP2/6-311G level of theory and their results are compared with the values obtained in this work.

In the present work, geometry optimizations of naphthalene dimers in various configurations-parallel-displaced (PD), parallel-displaced according to $C_{2 h}$ symmetry, crossed-parallel according to $C_{2}$ and $D_{2 h}$ symmetry-using the $\omega \mathrm{B} 97 \mathrm{X}-\mathrm{D}$ method with the $6-311 \mathrm{G}$ and $6-311 \mathrm{G}(\mathrm{p}, \mathrm{d})$ basis sets were performed. All stationary points were characterized by frequency calculations to ensure that the optimized structures were located at a local minimum. The basis-set superposition error (BSSE) correction developed by Simon et al. ${ }^{101}$ was included in the calculations for all complexes. Calculations without this correction were carried out to characterize the importance of this error. Negative binding energies indicate exothermic complex formation. ${ }^{102}$ Analysis of dimer configurations showed that only the low-level symmetry configurations, $\mathrm{C}_{2}$ and parallel-displaced (PD), represented in Figure $5 a$ and $b$, respectively, correspond to minima on the PES.

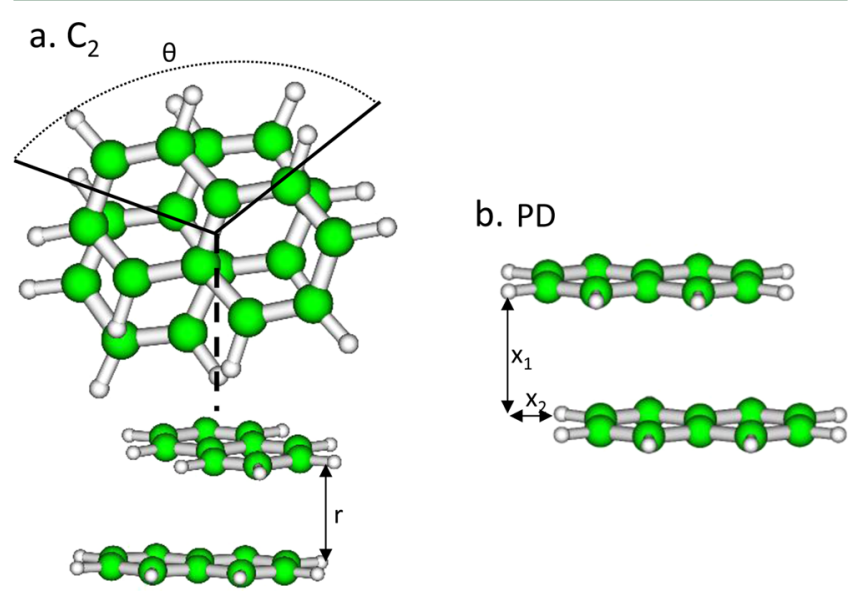

Figure 5. Optimized geometries at the $\omega \mathrm{B} 97 \mathrm{X}-\mathrm{D} / 6-311 \mathrm{G}$ level of theory for naphthalene dimers in the most stable configurations (a) C2 and (b) parallel-displaced (PD).

Vibrational calculations for the symmetric configurations exhibited negative frequencies. This outcome is in agreement with Walsh et al. ${ }^{99}$ who showed that structures of low symmetry are the most stable. The center of mass separation $(r)$, rotation angle $(\theta)$ for the $\mathrm{C}_{2}$ structure, and distance of relative translation $\left(x_{1}\right.$ and $\left.x_{2}\right)$ for the PD structure are given in Table 5. For these two configurations, the interaction energies

Table 5. Geometry Dependence of Basis Sets on Structures PD and C2 Shown in Figure 5

\begin{tabular}{lcccccc} 
& \multicolumn{2}{c}{$\mathrm{C}_{2}$} & & \multicolumn{3}{c}{$\mathrm{PD}$} \\
\cline { 2 - 3 } \cline { 6 - 7 } 6-311G & $\theta(\mathrm{deg})$ & $r(\AA)$ & & $r(\AA)$ & $x_{1}(\AA)$ & $x_{2}(\AA)$ \\
$+\mathrm{CP}^{a}$ & 129 & 3.38 & & 3.52 & 1.12 & 1.26 \\
6-311G** & 126 & 3.41 & & 3.44 & 1.08 & 1.29 \\
+ CP $^{a}$ & 130 & 3.45 & & 3.32 & 1.07 & 1.27 \\
cc-pVDZ+CP $^{a}$ & 133 & 3.43 & & 3.65 & 1.05 & 1.30 \\
Saeki et al. & 134 & 3.45 & & 3.49 & 1.11 & 1.28 \\
& 135 & 3.46 & & 3.40 & 1.03 & 1.31
\end{tabular}

${ }^{a} \mathrm{CP}$ refers to the keyword "counterpoise" used in Gaussian to take BSSE correction into account in the calculations.

at the different levels of theory considered, with and without BSSE correction, as well as the first harmonics, are reported in Table 6. Interaction energies and associated wavenumbers were obtained using $\omega$ B97X-D and 6-311G, 6-311G**, and ccpVDZ basis sets. Computed values are compared with results obtained by Walsh et al., ${ }^{99}$ Saeki et al., ${ }^{50}$ and Rubes et al. ${ }^{100}$ BSSE correction lowers the absolute value of the energy by 1.5 kcal.mol ${ }^{-1}$ on average, and represents only $0.04 \%$ of the binding energy. Thus, BSSE correction does not affect the 
Table 6. Interaction Energies in $\mathrm{kcal} / \mathrm{mol}$ and Associated Wavenumbers $\left(\mathrm{cm}^{-1}\right)$ of Optimized Naphthalene Dimers

\begin{tabular}{|c|c|c|c|c|c|}
\hline \multicolumn{2}{|c|}{ naphthalene } & \multicolumn{2}{|c|}{$\mathrm{C}_{2}$} & \multicolumn{2}{|c|}{$\mathrm{PD}$} \\
\hline \multirow[t]{2}{*}{$\begin{array}{l}\omega \mathrm{B} 97 \mathrm{X}-\mathrm{D} / 6-311 \mathrm{G} \\
\text { (this work) }\end{array}$} & $\begin{array}{l}\text { without } \\
\text { counterpoise }\end{array}$ & -9.00 & 14 & -8.72 & 30 \\
\hline & $\begin{array}{l}\text { with } \\
\text { counterpoise }\end{array}$ & -7.55 & 8 & -7.28 & 33 \\
\hline \multirow[t]{2}{*}{$\begin{array}{l}\omega \mathrm{B} 97 \mathrm{X}-\mathrm{D} / 6-311 \mathrm{G} * * \\
\text { (this work) }\end{array}$} & $\begin{array}{l}\text { without } \\
\text { counterpoise }\end{array}$ & -9.45 & 16 & -9.16 & 32 \\
\hline & $\begin{array}{l}\text { with } \\
\text { counterpoise }\end{array}$ & -7.86 & 12 & -7.58 & 29 \\
\hline $\begin{array}{l}\omega \mathrm{B} 97 \mathrm{X}-\mathrm{D} / \mathrm{cc}-\mathrm{pVDZ} \\
\text { (this work) }\end{array}$ & $\begin{array}{l}\text { with } \\
\text { counterpoise }\end{array}$ & -7.52 & 12 & -7.22 & 25 \\
\hline $\begin{array}{l}\mathrm{MP} 2 / 6-31 \mathrm{G}^{*}(0.25) \\
(\text { Walsh et al. } \\
\end{array}$ & $\begin{array}{l}\text { with } \\
\text { counterpoise }\end{array}$ & -8.15 & & -7.68 & \\
\hline \multirow[t]{2}{*}{$\begin{array}{l}\mathrm{MP} 2 / \mathrm{cc}-\mathrm{pVDZ} \\
\quad\left(\text { Saeki et al. }{ }^{50}\right)\end{array}$} & $\begin{array}{l}\text { without } \\
\text { counterpoise }\end{array}$ & -5.30 & 22 & -5.00 & 8 \\
\hline & $\begin{array}{l}\text { with } \\
\text { counterpoise }\end{array}$ & -6.24 & & -5.89 & \\
\hline $\begin{array}{l}\mathrm{DFT} / \mathrm{CCSD}(\mathrm{T}) \\
\quad\left(\text { Rubes et al. }{ }^{100}\right)\end{array}$ & & -6.23 & & -5.96 & \\
\hline $\begin{array}{l}\text { SAPT0/aug-cc-pVDZ } \\
\quad\left(\text { Hohenstein et al. }{ }^{103}\right)\end{array}$ & & & & -7.27 & \\
\hline
\end{tabular}

order of stability of the tested configurations. This outcome is in full agreement with the cited prior work. Further, there are no trends for the impact of basis set and BSSE correction on the geometry of the dimer configurations. However, by calculating the mean difference of computed values, this work, with those of Saeki et al., ${ }^{50}$ shows the 6-311G basis set combined with BSSE correction to be a preferred option.

Vibration Modes of Naphthalene Dimers. Wavenumber calculations for stable dimers were performed using the $\omega$ B97X-D/6-311G method with BSSE correction. From vibrational analysis of the monomer (Table S2 in the Supporting Information) and the dimer (Table 7), the vibrational modes arising from intra- and intermolecular modes can be discriminated. The assignments are reported in Table 7 for sets of calculations performed as part of this work and from Saeki et al. ${ }^{50}$ Calculated values are compared with experimental data when available. The calculations were performed with counterpoise correction for both the $\mathrm{C}_{2}$ and PD configurations. The calculated intra- and intermolecular wavenumbers, irrespective of calculation details, are readily distinguished for naphthalene. The intermolecular wavenumbers are at $\sim 100 \mathrm{~cm}^{-1}$ and lower, while the intramolecular wavenumbers are greater than $\sim 100 \mathrm{~cm}^{-1}$.

b. Pi-Stacking in the Acene Family. Optimization at the $\omega \mathrm{B} 97 \mathrm{XD} / 6-311 \mathrm{G}$ level with BSSE correction gives rise to the expected parallel tilted configuration ${ }^{105}$ for anthracene, tetracene, and pentacene dimers, as shown in Figure 6. This configuration closely resembles that found in triclinic tetracene $e^{54}$ and pentacene ${ }^{58}$ crystals. The associated interaction energies are reported Table 8 . Other configurations were found to be unstable. They possess negative calculated harmonic values and were clearly not located at a minimum of the PES. Thus, only naphthalene has a more energetically stable $\mathrm{C}_{2}$ configuration. Computations related to both the $\mathrm{C}_{2}$ and $\mathrm{PD}$ configurations for naphthalene are shown in Table 7. The PD configuration results are used for comparisons with other acene family members where it is the most stable configuration.

For a given geometry, in this case parallel-tilted, the binding interaction energy increases with the number of interacting cycles in a nonlinear manner. This result is consistent with previous studies by Hohenstein et al. ${ }^{103}$ and Grimme et al. ${ }^{106}$ who compared interaction energies of naphthenic and aromatic dimers of increasing size using the B2PLYP/TZV (2d,p) level of theory. They found that arene dimers were strongly preferred over equivalent naphthenic dimers, especially for anthracene and tetracene which were stabilized by $3-4 \mathrm{kcal} \cdot \mathrm{mol}^{-1}$. The calculations reported here are in accordance with these prior results and confirm the prevalence of $\pi$-stacking interactions.

The wavenumbers assigned to intermolecular (Table 9) and intramolecular (Table 10) modes of vibration for the acene family were calculated at the $\omega \mathrm{B} 97 \mathrm{XD} / 6-311 \mathrm{G}$ level of theory using BSSE correction. The characteristic wavenumbers for

Table 7. Calculated Wavenumber $\left(\mathrm{cm}^{-1}\right)$ of the Intra- and Intermolecular Vibrational Modes of the Naphthalene Dimer in Configurations $a$ and $b$ at the $\omega$ B97X-D/6311G Level with BSSE Correction, Compared with Frequencies Obtained at the $\mathrm{MP2} / \mathrm{cc}-\mathrm{pVDZ}$ Level in Saeki et al. ${ }^{50}$

\begin{tabular}{|c|c|c|c|c|c|c|}
\hline \multicolumn{2}{|c|}{$\begin{array}{l}\text { configuration } \mathrm{C}_{2} \\
\omega \mathrm{B} 97 \mathrm{X}-\mathrm{D} / 6-311 \mathrm{G}^{a}\end{array}$} & \multirow[b]{2}{*}{ Saeki et al. ${ }^{50} \mathrm{MP} 2 / \mathrm{cc}-\mathrm{pVDZ}{ }^{b}$} & \multicolumn{2}{|c|}{$\begin{array}{l}\text { configuration PD } \\
\omega \mathrm{B} 97 \mathrm{XD} / 6-311 \mathrm{G}^{a}\end{array}$} & \multirow[b]{2}{*}{ Saeki et al. ${ }^{50} \mathrm{MP} 2 / \mathrm{cc}-\mathrm{pVDZ}{ }^{b}$} & \multirow[b]{2}{*}{ experimental values ${ }^{104}$} \\
\hline harmonic & PT2 & & harmonic & PT2 & & \\
\hline \multicolumn{7}{|c|}{ Intramolecular } \\
\hline 184 & 181 & 185 & 188 & 181 & 180 & 176 \\
\hline 192 & 187 & 188 & 196 & 188 & 187 & 195 \\
\hline 199 & 198 & 203 & 207 & 201 & 201 & \\
\hline 203 & 202 & 205 & 208 & 201 & 207 & \\
\hline 378 & 370 & 351 & 380 & 372 & 351 & \\
\hline 378 & 371 & 351 & 381 & 372 & 351 & 359 \\
\hline 404 & 398 & 386 & 405 & 396 & 385 & \\
\hline 405 & 398 & 387 & 405 & 397 & 387 & \\
\hline \multicolumn{7}{|c|}{ Intermolecular } \\
\hline 14 & 16 & 22 & 33 & 29 & 8 & \\
\hline 32 & 34 & 45 & 36 & 35 & 39 & \\
\hline 50 & 50 & 64 & 55 & 51 & 54 & \\
\hline 78 & 83 & 95 & 75 & 68 & 81 & \\
\hline 85 & 89 & 103 & 88 & 85 & 100 & \\
\hline 94 & 99 & 106 & 101 & 98 & 110 & \\
\hline
\end{tabular}

${ }^{a}$ With counterpoise correction. ${ }^{b}$ Without counterpoise correction. 

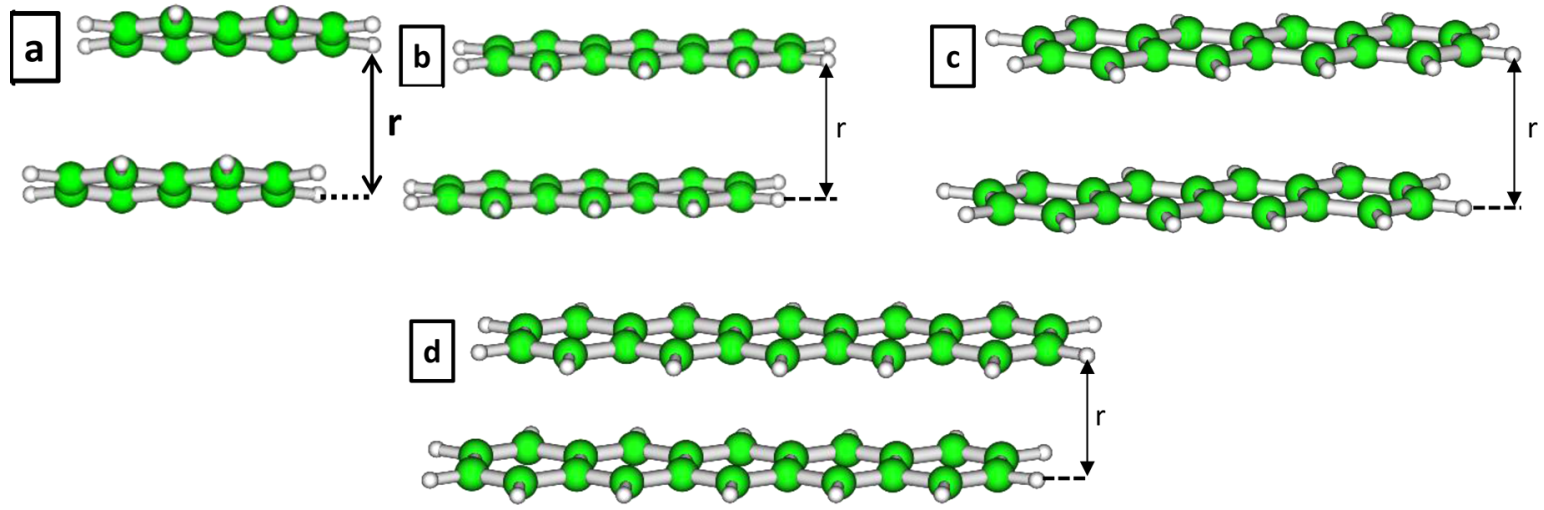

Figure 6. Acene dimers in the parallel-displaced (PD) configuration: (a) naphthalene; (b) anthracene; (c) tetracene; (d) pentacene.

Table 8. Intermolecular Distance and Interaction Energies Calculated for Acene Dimers

\begin{tabular}{|c|c|c|c|c|c|}
\hline & dimers & naphthalene & anthracene & tetracene & pentacene \\
\hline intermolecular distance $r(\AA)$ & & 3.41 & 3.27 & 2.96 & 2.98 \\
\hline \multirow[t]{2}{*}{ interaction energies $(\mathrm{kcal} / \mathrm{mol})$} & $\omega \mathrm{B} 97 \mathrm{XD} / 6-311 \mathrm{G}+\mathrm{BSSE}$ correction & -7.53 & -11.52 & -15.73 & -20.44 \\
\hline & Hohenstein et al. ${ }^{101}$ SAPT0/aug-cc-pVDZ & -7.27 & -12.24 & -17.49 & -22.91 \\
\hline
\end{tabular}

Table 9. Calculated Intermolecular Vibrational Modes $\left(\mathrm{cm}^{-1}\right)$ and Their Intensities for Naphthalene, Anthracene, Tetracene, and Pentacene Dimers

\begin{tabular}{lllll}
\multicolumn{1}{c}{ type $^{a}$} & naphthalene & anthracene & tetracene & pentacene \\
rotation $1 / 2$ & $33(0.04)$ & $22(0.004)$ & $15(0.002)$ & $19(0.002)$ \\
translation $1 / 2$ & $36(0)$ & $34(0)$ & $31(0)$ & $27(0)$ \\
total rotation + & $55(0)$ & $57(0)$ & $52(0)$ & $57(0)$ \\
$\quad$ translation $1 / 2$ & & & & \\
& $75(0.21)$ & $75(0.05)$ & $87(0.14)$ & $74(0.07)$ \\
rotation $1 / 2$ & $88(0.08)$ & $87(0.8)$ & $80(0.96)$ & $72(0.9)$ \\
& $101(0)$ & $111(0.8) /$ & $112(0)$ & $95(0)$
\end{tabular}

${ }^{a} 1 / 2$ means one molecule relative to the other one.

intermolecular vibrations fall in the same range for all acene dimers. The lower bound wavenumbers are $15-33 \mathrm{~cm}^{-1}$, and the upper bound wavenumbers are $94-112 \mathrm{~cm}^{-1}$. The wavenumbers for the intramolecular vibrations are found at higher wavenumbers but begin to overlap with the intermolecular vibration wavenumbers starting with anthracene where the intermolecular mode at a wavenumber of $111 \mathrm{~cm}^{-1}$ with an intensity of 0.8 is not readily discriminated from the intramolecular mode at $112 \mathrm{~cm}^{-1}$ with an intensity of 1.2 , on the basis of wavenumber or intensity value. Thus, inter- and intramolecular vibrations are easily discriminated for naphthalene, and the inter- and intramolecular vibration modes are uncorrelated. For anthracene, tetracene, and pentacene, the lowest intramolecular vibration modes fall within the range of intermolecular vibrations.

Four vibration modes, given in Table 9, corresponding to rotation, translation, rotation of the dimer combined with translation and rotation movements of one molecule relative to the other, are common for all four dimers. The last two intermolecular modes are compound-specific. For tetracene, the two additional modes comprise twisting movements of molecular pairs in the same direction $\left(87 \mathrm{~cm}^{-1}\right)$ and in opposite directions $\left(112 \mathrm{~cm}^{-1}\right)$. For pentacene, one is a wagging movement $\left(74 \mathrm{~cm}^{-1}\right)$ and the other comprises an opposite direction wagging movement combined with vertical translation normal to the two molecules $\left(95 \mathrm{~cm}^{-1}\right)$. For anthracene, one mode is a rotation of one molecule relative to the other, and the second is split into two modes. These latter two modes, at 111 and $112 \mathrm{~cm}^{-1}$, consist of a butterfly movement by one molecule with a half-twisting movement by the other simultaneously.

The other vibration modes, shown in Table 10, correspond to intramolecular vibrations. For every type of vibration, simultaneous vibration of each molecule is observed, either in the same or opposite direction, resulting in two wavenumbers. These alternating motions are characterized by wavenumbers and intensities that depend on the value of the dipole moment. The butterfly vibration mode presents very different wavenumbers depending on whether the molecular movements are in the same or opposite direction. Differences range in value from 8 to $42 \mathrm{~cm}^{-1}$. The impact of relative movement increases with the number of aromatic rings, due to the correlation with twisting modes at higher frequencies.

Trends in Values for Specific Bands in the Acene Family. In order to visualize the effect of the number of aromatic rings on the wavenumbers of specific vibration modes, intramolecular vibration modes and intermolecular vibrations are presented in Figures 7 and 8, respectively. Intramolecular vibration modes shift to lower wavenumbers with increasing molecule size irrespective of the vibrational mode considered, as was observed for monomers. Butterfly (B), twisting $(\tau)$, wagging $(\omega)$, stretching $(\sigma)$, and rocking $(\rho)$ movements behave similarly. By contrast, the intermolecular vibration modes, found at similar wavenumbers for all the molecules in the acene family, appear specific to this family, and may be used as a reliable fingerprint. In addition, twisting vibration bands appear for dimers of tetracene at $208 \mathrm{~cm}^{-1}$ and pentacene at $168 \mathrm{~cm}^{-1}$ that replace corresponding twisting bands for monomers at 154 and $104 \mathrm{~cm}^{-1}$. The absence of a mode at $154 \mathrm{~cm}^{-1}$ for tetracene dimers supports the hypothesis that the feature observed experimentally at $142 \mathrm{~cm}^{-1}$ for monomers (see Table 3) is an overtone.

The Impact of Molecule Distortion on the Vibration Modes of Acene Dimers. The impact of molecular distortion on vibration modes for dimers was evaluated in the same manner as for monomers. The molecular conformations were 
Table 10. Intramolecular Vibration Modes $\left(\mathrm{cm}^{-1}\right)$ and the Corresponding Intensities in Brackets $(\mathrm{km} / \mathrm{mol})$ for Naphthalene, Anthracene, Tetracene, and Pentacene Dimers ${ }^{a}$

\begin{tabular}{|c|c|c|c|c|}
\hline type $^{b}$ & naphthalene & anthracene & tetracene & pentacene \\
\hline butterfly sw & $\begin{array}{l}188(3.8) \\
177(3.2)\end{array}$ & $\begin{array}{l}111(0.8) / 112(1.2) \\
93(1.5)\end{array}$ & $\begin{array}{l}59(0.6) \\
57(0.8)\end{array}$ & $\begin{array}{l}42(0.4) \\
38(0.5)\end{array}$ \\
\hline butterfly ow & $\begin{array}{l}196(0) \\
177(3.2)\end{array}$ & $\begin{array}{l}128(0) \\
93(1.5)\end{array}$ & $\begin{array}{l}94(0) \\
57(0.8)\end{array}$ & $\begin{array}{l}84(0) \\
38(0.5)\end{array}$ \\
\hline twisting sw & $\begin{array}{l}207(1.2) \\
191(0)\end{array}$ & $\begin{array}{l}148(0.6) \\
124(0)\end{array}$ & $\begin{array}{l}117(0.3) \\
93(0)\end{array}$ & $\begin{array}{l}108(0.2) \\
74(0)\end{array}$ \\
\hline twisting ow & $\begin{array}{l}208(0) \\
191(0)\end{array}$ & $\begin{array}{l}149(0.02) \\
124(0)\end{array}$ & $\begin{array}{l}124(0) \\
93(0)\end{array}$ & $\begin{array}{l}101(0.002) \\
74(0)\end{array}$ \\
\hline scissoring sw & $\begin{array}{l}381(2.2) \\
379(1.5)\end{array}$ & $\begin{array}{l}244(2.1) \\
243(1.4)\end{array}$ & $\begin{array}{l}168(1.7) \\
168(1.3)\end{array}$ & $\begin{array}{l}123(1.5) \\
123(1.0)\end{array}$ \\
\hline scissoring ow & $\begin{array}{l}380(0) \\
379(1.5)\end{array}$ & $\begin{array}{l}243(0) \\
243(1.4)\end{array}$ & $\begin{array}{l}169(0) \\
168(1.3)\end{array}$ & $\begin{array}{l}125(0) \\
123(1.0)\end{array}$ \\
\hline wagging sw & $\begin{array}{l}405(0) \\
403(0)\end{array}$ & $\begin{array}{l}250(0) \\
276(1.1)\end{array}$ & $\begin{array}{l}162(0) \\
196(0)\end{array}$ & $116(0) 150(0)$ \\
\hline wagging ow & $\begin{array}{l}405(0.02) \\
403(0)\end{array}$ & $\begin{array}{l}253(0.2) \\
276(1.1)\end{array}$ & $\begin{array}{l}176(0.2) \\
196(0)\end{array}$ & $\begin{array}{l}130(0.08) \\
150(0)\end{array}$ \\
\hline twisting sw & $\begin{array}{l}498(0) \\
494(0)\end{array}$ & $\begin{array}{l}285(0.02) \\
281(0)\end{array}$ & $208(0)$ & $168(0.05)$ \\
\hline twisting ow & $\begin{array}{l}499(15.2) \\
494(0)\end{array}$ & $\begin{array}{l}286(0.08) \\
281(0)\end{array}$ & $212(0.09)$ & $168(0.2)$ \\
\hline wagging sw & $\begin{array}{l}504(45.0) \\
504(28.7)\end{array}$ & $\begin{array}{l}396(0.2) \\
394(0.05)\end{array}$ & $\begin{array}{l}282(1.6) \\
276(1.1)\end{array}$ & $\begin{array}{l}204(2.2) \\
198(1.3)\end{array}$ \\
\hline wagging ow & $\begin{array}{l}507(0) \\
504(28.7)\end{array}$ & $\begin{array}{l}396(0.04) \\
394(0.05)\end{array}$ & $\begin{array}{l}286(0) \\
276(1.1)\end{array}$ & $\begin{array}{l}210(0.002) \\
198(1.3)\end{array}$ \\
\hline stretching sw & $\begin{array}{l}534(0) \\
534(0)\end{array}$ & $\begin{array}{l}407(0) \\
407(0)\end{array}$ & $\begin{array}{l}324(0) \\
324(0)\end{array}$ & $\begin{array}{l}269(0) \\
269(0)\end{array}$ \\
\hline stretching ow & $\begin{array}{l}534(0.09) \\
534(0)\end{array}$ & $\begin{array}{l}407(0.04) \\
407(0)\end{array}$ & $\begin{array}{l}325(0.06) \\
324(0)\end{array}$ & $\begin{array}{l}269(0.05) \\
269(0)\end{array}$ \\
\hline rocking sw & $\begin{array}{l}537(0) \\
537(0)\end{array}$ & $\begin{array}{l}412(0) \\
412(0)\end{array}$ & $\begin{array}{l}316(0) \\
316(0)\end{array}$ & $\begin{array}{l}246(0) \\
247(0)\end{array}$ \\
\hline rocking ow & $\begin{array}{l}537(0.06) \\
537(0)\end{array}$ & $\begin{array}{l}412(0.01) \\
412(0)\end{array}$ & $\begin{array}{l}316(0.03) \\
316(0)\end{array}$ & $\begin{array}{l}247(0.05) \\
247(0)\end{array}$ \\
\hline twisting sw & $\begin{array}{l}658(0.4) \\
656(0)\end{array}$ & & $\begin{array}{l}335(0.03) \\
327(0)\end{array}$ & $\begin{array}{l}256(0.02) \\
247(0)\end{array}$ \\
\hline twisting ow & $\begin{array}{l}659(0) \\
656(0)\end{array}$ & & $\begin{array}{l}336(0) \\
327(0)\end{array}$ & $\begin{array}{l}257(0.008) \\
247(0)\end{array}$ \\
\hline wagging sw & & $\begin{array}{l}496(70.5) \\
495(0)\end{array}$ & $\begin{array}{l}390(0) \\
389(0)\end{array}$ & $\begin{array}{l}302(0.005) \\
247(0)\end{array}$ \\
\hline wagging ow & & $\begin{array}{l}497(0.5) \\
495(0)\end{array}$ & $\begin{array}{l}390(0.02) \\
389(0)\end{array}$ & $\begin{array}{l}303(0.07) \\
247(0)\end{array}$ \\
\hline
\end{tabular}

${ }^{a}$ Values of corresponding monomer modes are written below in italics. ${ }^{b}$ sw $=$ same way (i.e., resulting dipole moment in the same direction for the two molecules). ow = opposite way (i.e., resulting dipole moment in opposite directions for the molecules).

perturbed as illustrated in Figure 3, and the distance $r$ (defined in Figure 6) between the molecules was set equal to 3.4, 3.4, and $2.9 \AA$ for naphthalene, anthracene, and tetracene dimers, respectively. As distortion reduces the aromaticity of the molecules, the stability of $\pi$-stacked dimers is expected to be reduced or eliminated. Table 11, where interaction energies for these systems are listed, highlights this loss of stability. For example, no conformations for distorted pentacene dimers correspond to local minima in the PES. Distortion did not affect intramolecular modes of vibration overall for dimers, as shown in Table S3 in the Supporting Information. Only butterfly vibration modes for anthracene and tetracene, whose wavenumbers get closer to the ones observed for monomers, shown in Table 10, appear to be affected. Some other modes, for which two wavenumbers are given in Table S3 (Supporting Information), do not exhibit the combined movement of the two molecules either in the same direction or in the opposite direction (as observed for the other intramolecular modes) but movement of the molecules simultaneously. These exceptions are a consequence of the decoupling between the molecules forming the distorted dimer. However, the intermolecular vibration modes, shown in Table 12, are shifted to lower wavenumbers compared to the symmetric analogues shown in Table 9. For the symmetric dimers, the intermolecular vibration modes are effectively independent of molecule size, while, for the distorted molecules, the vibration modes tend to lower values as molecular size increases.

The behavior of the symmetric dimers is attributed to a balancing of opposing effects. One would expect intermolecular vibration modes to shift to lower wavenumbers with increasing ring number (as is observed for intramolecular modes). However, the intensity of the intermolecular interactions increases with the number of rings (Table 8) for symmetric dimers, and this tends to increase wavenumbers. This compensative effect is diminished or absent for the distorted dimers; hence, the difference in behavior and wavenumber invariance for a given intermolecular vibration mode may be a characteristic of $\pi$-stacking interactions.

Identification of Unassigned Peaks in the Experimental Spectra of Tetracene and Pentacene. By subtracting the intermolecular wavenumbers from the symmetric dimer vibrational spectra of tetracene and pentacene, and comparing the resultant spectra with the spectra of the corresponding monomers, there are two peaks, one for tetracene at $117 \mathrm{~cm}^{-1}$ 


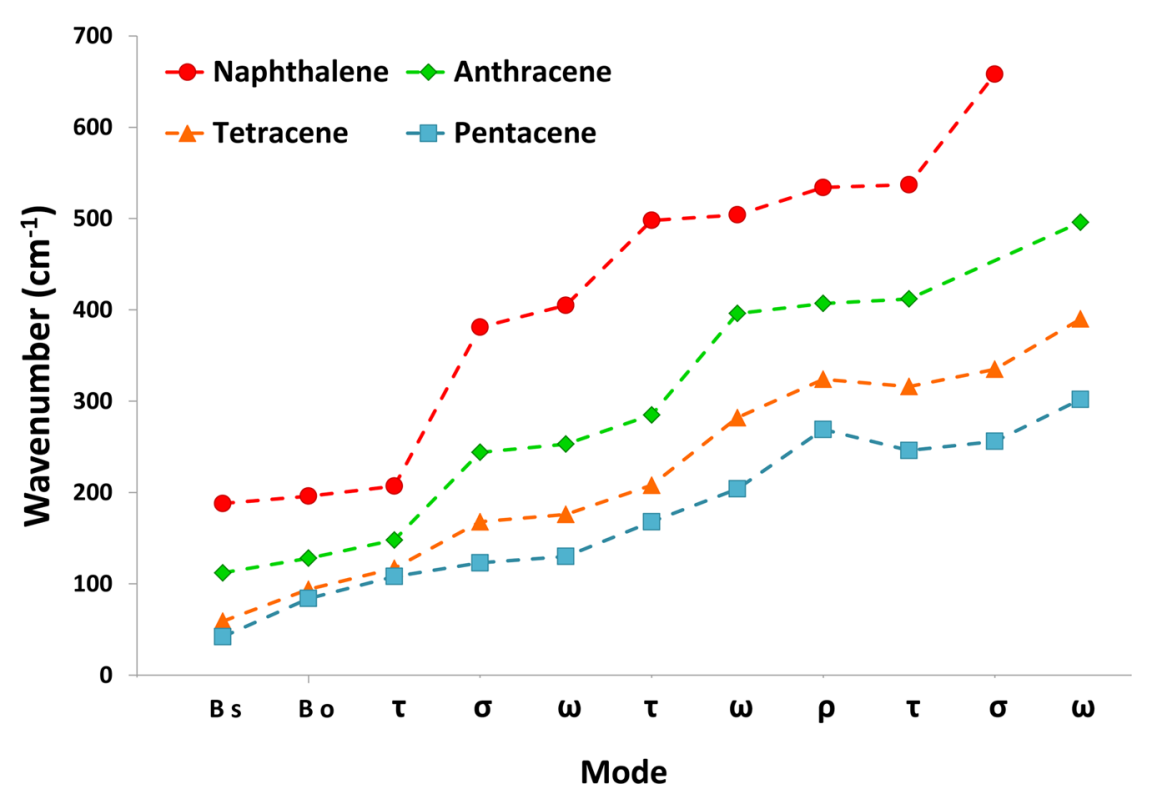

Figure 7. Comparison of theoretical intramolecular vibration modes for naphtalene, anthracene, tetracene, and pentacene dimers: B s and B o designate butterfly modes, same (s) and opposite (o) direction, respectively; $\tau, \sigma, \omega$, and $\rho$ take place for twisting, stretching, wagging, and rocking modes, respectively.

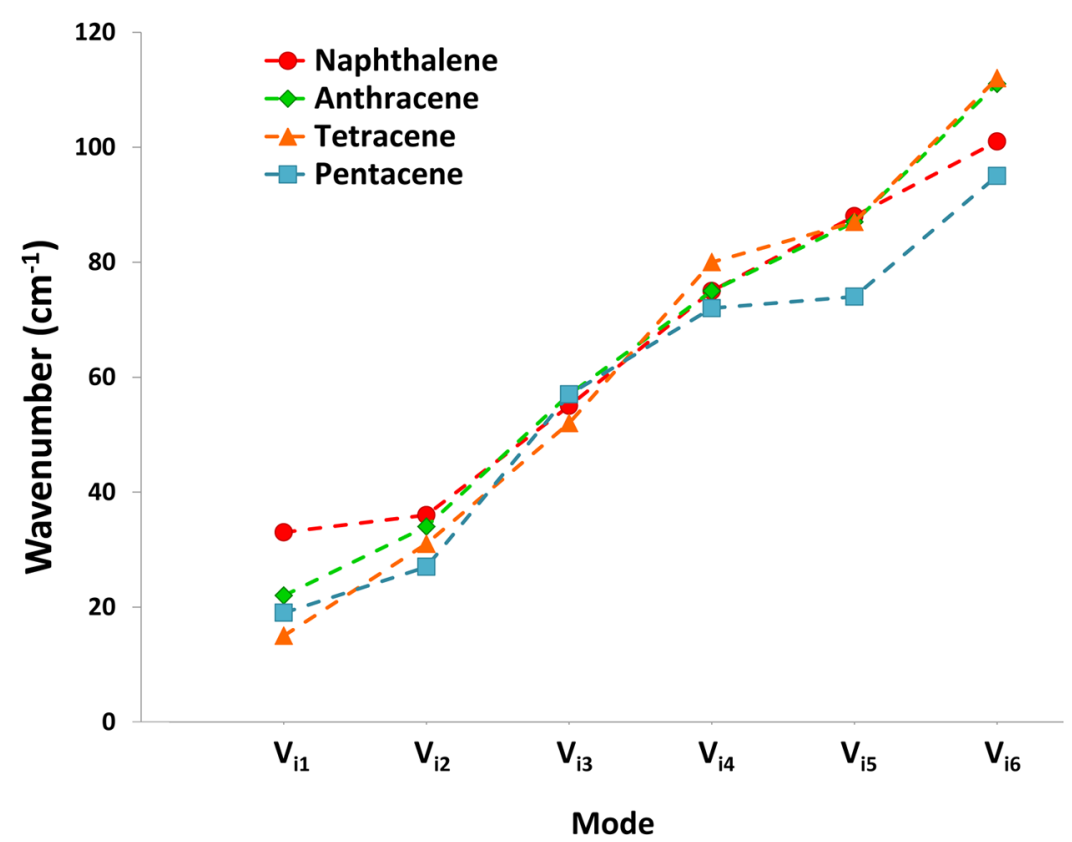

Figure 8. Comparison of theoretical intermolecular vibration modes for naphthalene, anthracene, tetracene, and pentacene dimers.

\section{Table 11. Interaction Energies Calculated for Distorted Acene Dimers ${ }^{a}$}

$\begin{array}{lccc} & \text { dimers } & \text { naphthalene } & \text { anthracene } \\ \text { interaction energies }(\mathrm{kcal} / \mathrm{mol}) & \omega \mathrm{B} 97 \mathrm{XD} / 6-311 \mathrm{G}+\mathrm{BSSE} \text { correction } & +1.23(+8.76) & -4.27(+7.25) \\ { } \text { The values in parentheses indicate the energy difference between the interaction energy of distorted and symmetric acene dimers. }\end{array}$

and one for pentacene at $108 \mathrm{~cm}^{-1}$, with intensities in the same order of magnitude as the ones calculated for fundamental bands for tetracene $(0.34 \mathrm{~km} / \mathrm{mol})$ and pentacene $(0.15 \mathrm{~km} /$ $\mathrm{mol})$. These peaks arise because, for every vibrational mode occurring for monomers, there are two quasi-degenerate modes arising for dimers depending on whether the molecules vibrate in the same or opposite direction. Thus, modes that are Raman- active for a monomer become infrared-active for dimers vibrating in opposite directions.

With this final set of assignments, the intermolecular and intramolecular vibration analysis for the acene family of compounds is complete. Figure 9 provides a direct comparison of experimental and calculated spectra with the finalized assignments. There are no residual unassigned peaks in the available experimental spectra, and ultra-low-wavenumber 
Table 12. Calculated Intermolecular Vibrational Modes $\left(\mathrm{cm}^{-1}\right)$ and Their Intensities for Distorted Dimers of Naphthalene, Anthracene, and Tetracene

\begin{tabular}{llll}
\multicolumn{1}{c}{ type $^{a}$} & naphthalene & anthracene & tetracene \\
translation $1 / 2$ & $12(0.015)$ & $11(0.004)$ & $6(0)$ \\
rotation $1 / 2$ & $32(0.04)$ & $21(0.004)$ & $13(0.004)$ \\
translation $1 / 2$ & $47(0.007)$ & $33(0.002)$ & $21(0.005)$ \\
rotation + translation & $60(0.006)$ & $41(0.009)$ & $33(0.003)$ \\
translation (remoteness of & $73(0.008)$ & $55(0.01)$ & $46(0.2)$ \\
$\quad$ molecules) & & & \\
rotation $1 / 2$ & $94(0.14)$ & $73(0.2)$ & \\
twisting of the full system & & & $74(0.08)$ \\
$a_{1 / 2}$ man & & &
\end{tabular}

${ }^{a} 1 / 2$ means one molecule relative to the other.

intermolecular peaks are hypothesized. While these have yet to be measured experimentally, identification and discrimination of intermolecular and intramolecular bands at low wavenumbers will play a more important role in the future as the range of IR measurements broadens. For example, by using coherent synchrotron radiation, it is becoming possible to make experimental photoacoustic infrared measurements in the range 7-30 $\mathrm{cm}^{-1}$, as demonstrated recently by Billinghurst and Michaelian. ${ }^{107}$ DFT calculations have much to contribute to this development, and the interpretation of results obtained.

\section{CONCLUSIONS}

The DFT calculations presented in this work provide detailed and accurate descriptions of experimental photoacoustic farinfrared spectra for tetracene and pentacene and for far-infrared spectra of the acene family as a whole. The impacts of molecular distortion on monomer and dimer spectra and dimer stability and the interpretation of peaks in photoacoustic infrared spectra of crystalline solids are discussed in detail. Distortion breaks molecular symmetry, and this adds to the complexity of the interpretation of the resulting spectra. Intermolecular and intramolecular vibrations in the acene family of compounds are discriminated, and combinations of intermolecular and intramolecular vibrations are identified. Peak assignment ambiguity and the potential impacts of molecular distortion on peak intensity are addressed for tetracene and pentacene. By examining computed and experimental spectra for the family of acene molecules concurrently, trends in vibration modes with molecular size were used to reinforce assignments, and to reattribute assignments made previously in the experimental tetracene and pentacene spectra. In particular, dimer calculations permitted reassignment of an infrared-active peak arising in tetracene at $142 \mathrm{~cm}^{-1}$ as an overtone, and assignment of large infrared-active peaks at 117 and $108 \mathrm{~cm}^{-1}$ for tetracene and pentacene, respectively, that may permit their identification in an acene mixture.

The intermolecular vibrations for naphthalene, anthracene, tetracene, and pentacene are found in the same wavelength range of the far-infrared spectrum and are not readily discriminated one from the other. The specificity of the acene $\pi$-stacking interactions, reinforced by calculations with distorted dimers, not only allows the identification of this family of molecules but also highlights the presence of $\pi$-stacking interaction in a mixture as a whole.

\section{ASSOCIATED CONTENT}

\section{S Supporting Information}

Detailed aspects of tetracene (molecule) geometry and computed outcomes referred to in the text as Tables S1-S3

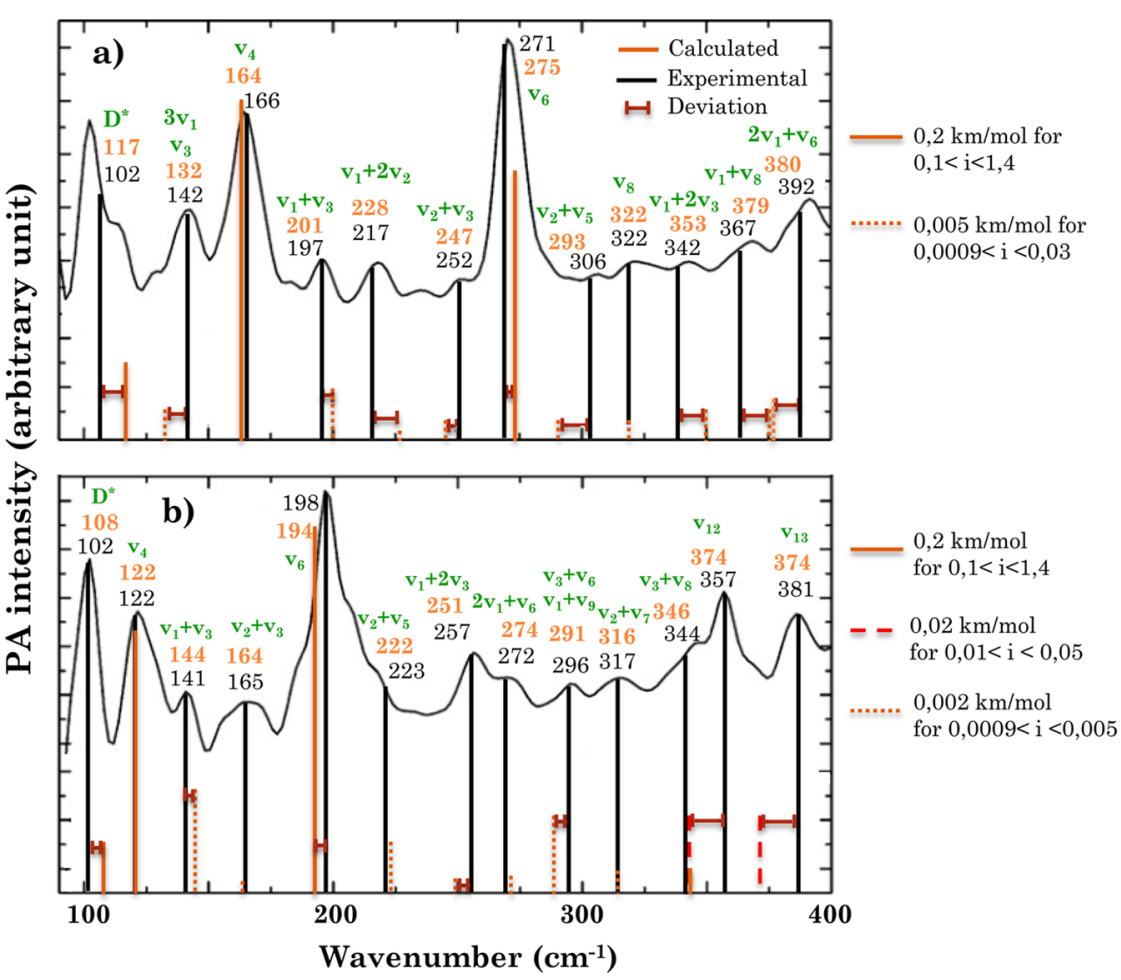

Figure 9. Final assignments of the experimental infrared transitions for (a) tetracene and (b) pentacene. Calculated wavenumbers are indicated on the spectra with orange lines, with corresponding values above. Experimental values are indicated for comparison, and represented with dark lines. Computed intensities are scaled in order to provide qualitative information. 
and Figures S1 and S2. This material is available free of charge via the Internet at http://pubs.acs.org.

\section{AUTHOR INFORMATION}

\section{Corresponding Author}

*E-mail: jmshaw@ualberta.ca.

\section{Notes}

The authors declare no competing financial interest.

\section{ACKNOWLEDGMENTS}

The authors thank Dr. K. Michaelian for generously sharing unpublished details of his experiments and results. One of the authors (F.S.) thanks Alain Dargelos for constant encouragement, support, and help. Computer time for this study was provided by the MCIA (Mésocentre de Calcul Intensif Aquitain) at the Université de Bordeaux and at the Université de Pau et des Pays de l'Adour. Funding provided by the sponsors of the NSERC Industrial Research Chair in Petroleum Thermodynamics (Natural Sciences and Engineering Research Council of Canada (NSERC), Alberta Innovates Energy and Environment Solutions, British Petroleum, ConocoPhillips Canada, Nexen Inc., Shell Canada Ltd., Total E\&P Canada Ltd., Virtual Materials Group), is gratefully acknowledged.

\section{REFERENCES}

(1) Lumpkin, H. E.; Johnson, B. H. Identification of Compound Types in Heavy Petroleum Gas Oil. Anal. Chem. 1954, 26, 1719-1722.

(2) Drushel, H. V.; Sommers, A. L. Isolation and Identification of Nitrogen Compounds in Petroleum. Anal. Chem. 1966, 38, 19-28.

(3) Damsté, J. S. S.; et al. The occurrence and identification of series of organic sulphur compounds in oils and sediment extracts. I. A study of Rozel Point Oil (U.S.A.). Geochim. Cosmochim. Acta 1987, 51, 2369-2391.

(4) Wang, Z.; Fingas, M.; Li, K. Fractionation of a Light Crude Oil and Identification and Quantitation of Aliphatic, Aromatic, and Biomarker Compounds by GC-FID and GC-MS, Part II. J. Chromatogr. Sci. 1994, 32, 367-382.

(5) Guan, S.; Marshall, A. G.; Scheppele, S. E. Resolution and Chemical Formula Identification of Aromatic Hydrocarbons and Aromatic Compounds Containing Sulfur, Nitrogen, or Oxygen in Petroleum Distillates and Refinery Streams. Anal. Chem. 1996, 68, 4671.

(6) Rudzinski, W. E.; Aminabhavi, T. M. A Review on Extraction and Identification of Crude Oil and Related Products Using Supercritical Fluid Technology. Energy Fuels 2000, 14, 464-475.

(7) Qian, K.; et al. Resolution and Identification of Elemental Compositions for More than 3000 Crude Acids in Heavy Petroleum by Negative-Ion Microelectrospray High-Field Fourier Transform Ion Cyclotron Resonance Mass Spectrometry. Energy Fuels 2001, 15, $1505-1511$.

(8) Hughey, C. A.; Rodgers, R. P.; Marshall, A. G.; Qian, K.; Robbins, W. K. Identification of acidic NSO compounds in crude oils of different geochemical origins by negative ion electrospray Fourier transform ion cyclotron resonance mass spectrometry. Org. Geochem. 2002, 33, 743-759.

(9) Wang, C.; Xu, J.; Weiss, R. G. Factors Influencing Orientations of Covalently-Attached and Doped Aromatic Groups in Stretched Polyethylene Films. J. Phys. Chem. B 2003, 107, 7015-7025.

(10) Speight, J. G. The Chemistry and Technology of Petroleum, 4th ed.; CRC Press: Boca Raton, FL, 2006.

(11) Kowalewski, I.; et al. Formation of $\mathrm{H} 2 \mathrm{~S}$ and sulfur-rich bitumen from a reservoired heavy oil in the presence of elemental sulfur. Org. Geochem. 2010, 41, 951-958.

(12) Podgorski, D. C.; et al. Heavy petroleum composition. 5. Compositional and structural continuum of petroleum revealed. Energy Fuels 2013, 27, 1268-1276.
(13) Zhurov, K. O.; Kozhinov, A. N.; Tsybin, Y. O. Hexagonal class representation for fingerprinting and facile comparison of petroleomic samples. Anal. Chem. 2013, 85, 5311-5315.

(14) Andrews, A. B.; et al. Molecular Orientation of Asphaltenes and $\mathrm{PAH}$ Model Compounds in Langmuir-Blodgett Films Using Sum Frequency Generation Spectroscopy. Langmuir 2011, 27, 6049-6058.

(15) Mullins, O. C.; Seifert, D. J.; Zuo, J. Y.; Zeybek, M. Clusters of Asphaltene Nanoaggregates Observed in Oilfield Reservoirs. Energy Fuels 2013, 27, 1752-1761.

(16) Vogel, R.; Siebert, F. Vibrational spectroscopy as a tool for probing protein function. Curr. Opin. Chem. Biol. 2000, 4, 518-523.

(17) Bellisola, G.; Sorio, C. Infrared spectroscopy and microscopy in cancer research and diagnosis. Am. J. Cancer Res. 2011, 2, 1-21.

(18) Remorino, A.; Hochstrasser, R. M. Three-Dimensional Structures by Two-Dimensional Vibrational Spectroscopy. Acc. Chem. Res. 2012, 45, 1896-1905.

(19) Burling-Claridge, R. Quantifying Meat Properties Using NearInfrared Spectroscopy. Handbook of Vibrational Spectroscopy; John Wiley \& Sons, Ltd: 2010 (http://onlinelibrary.wiley.com/doi/10.1002/ 0470027320.s8956/abstract).

(20) Cozzolino, D.; Cynkar, W. U.; Shah, N.; Smith, P. A. Can spectroscopy geographically classify Sauvignon Blanc wines from Australia and New Zealand? Food Chem. 2011, 126, 673-678.

(21) Cozzolino, D. Recent Trends on the Use of Infrared Spectroscopy to Trace and Authenticate Natural and Agricultural Food Products. Appl. Spectrosc. Rev. 2012, 47, 518-530.

(22) Dopfer, O. Laboratory Spectroscopy of Protonated PAH Molecules Relevant For Interstellar Chemistry. EAS Publ. Ser. 2011, 46, 103-108.

(23) Begue, D.; Pouchan, C.; Guillemin, J.-C.; Benidar, A. Anharmonic treatment of vibrational resonance polyads-the diborane: a critical case for numerical methods. Theor. Chem. Acc. 2012, 131, 1-11.

(24) Wustholz, K. L.; et al. Structure-Activity Relationships in Gold Nanoparticle Dimers and Trimers for Surface-Enhanced Raman Spectroscopy. J. Am. Chem. Soc. 2010, 132, 10903-10910.

(25) Wei, X.; Hong, S.-C.; Lvovsky, A. I.; Held, H.; Shen, Y. R. Evaluation of Surface vs Bulk Contributions in Sum-Frequency Vibrational Spectroscopy Using Reflection and Transmission Geometries. J. Phys. Chem. B 2000, 104, 3349-3354.

(26) Brixner, T.; et al. Two-dimensional spectroscopy of electronic couplings in photosynthesis. Nature 2005, 434, 625-628.

(27) Nielsen, H. H. The Vibration-Rotation Energies of Molecules. Rev. Mod. Phys. 1951, 23, 90-136.

(28) Bowman, J. M. Self-consistent field energies and wavefunctions for coupled oscillators. J. Chem. Phys. 1978, 68, 608-610.

(29) Gerber, R. B.; Ratner, M. A. A semiclassical self-consistent field (SC SCF) approximation for eigenvalues of coupled-vibration systems. Chem. Phys. Lett. 1979, 68, 195-198.

(30) Mills, I. M.; Robiette, A. G. On the relationship of normal modes to local modes in molecular vibrations. Mol. Phys. 1985, 56, 743-765.

(31) Bowman, J. M. The self-consistent-field approach to polyatomic vibrations. Acc. Chem. Res. 1986, 19, 202-208.

(32) Gerber, R. B.; Ratner, M. A. Mean-field models for molecular states and dynamics: new developments. J. Phys. Chem. 1988, 92, $3252-3260$

(33) Lehmann, K. K. Beyond the $x-K$ relations. Calculations of $1-1$ and 2 - 2 resonance constants with applications to HCN and DCN. Mol. Phys. 1992, 75, 739-739.

(34) Borro, A. F.; Mills, I. M.; Venuti, E. Quartic anharmonic resonances in acetylenes and haloacetylenes. J. Chem. Phys. 1995, 102, $3938-3944$.

(35) Wyatt, R. E.; Iung, C.; Leforestier, C. Toward ab Initio Intramolecular Dynamics. Acc. Chem. Res. 1995, 28, 423-429.

(36) Jung, J. O.; Gerber, R. B. Vibrational wave functions and spectroscopy of $(\mathrm{H} 2 \mathrm{O}) \mathrm{n}, \mathrm{n}=2,3,4,5$ : Vibrational self-consistent field with correlation corrections. J. Chem. Phys. 1996, 105, 10332-10348. 
(37) Norris, L. S.; Ratner, M. A.; Roitberg, A. E.; Gerber, R. B. Mo/ ller-Plesset perturbation theory applied to vibrational problems. $J$. Chem. Phys. 1996, 105, 11261-11267.

(38) Martin, J. M. L.; Taylor, P. R. Accurate ab initio quartic force field for trans-HNNH and treatment of resonance polyads. Spectrochim. Acta, Part A 1997, 53, 1039-1050.

(39) Wang, X.-G.; Edwin, L. Sibert. A nine-dimensional perturbative treatment of the vibrations of methane and its isotopomers. J. Chem. Phys. 1999, 111, 4510-4522.

(40) Barone, V. Anharmonic vibrational properties by a fully automated second-order perturbative approach. J. Chem. Phys. 2005, 122,014108

(41) Kjaergaard, H. G.; et al. Calculation of Vibrational Transition Frequencies and Intensities in Water Dimer: Comparison of Different Vibrational Approaches. J. Phys. Chem. A 2008, 112, 4324-4335.

(42) Carter, S.; Handy, N. C.; Bowman, J. M. High torsional vibrational energies of $\mathrm{H} 2 \mathrm{O} 2$ and $\mathrm{CH} 3 \mathrm{OH}$ studied by MULTIMODE with a large amplitude motion coupled to two effective contraction schemes. Mol. Phys. 2009, 107, 727-737.

(43) Seidler, P.; Christiansen, O. Automatic derivation and evaluation of vibrational coupled cluster theory equations. J. Chem. Phys. 2009, 131, 234109-1-234109-15

(44) Wong, M. W. Vibrational frequency prediction using density functional theory. Chem. Phys. Lett. 1996, 256, 391-399.

(45) Ammundsen, B.; Burns, G. R.; Islam, M. S.; Kanoh, H.; Rozière, J. Lattice Dynamics and Vibrational Spectra of Lithium Manganese Oxides: A Computer Simulation and Spectroscopic Study. J. Phys. Chem. B 1999, 103, 5175-5180.

(46) Hirata, S.; et al. In Recent Advances in Spectroscopy; Chaudhuri, R. K., Mekkaden, M. V., Raveendran, A. V., Narayanan, A. S.; Springer: Berlin, Heidelberg, 2010; pp 21-30.

(47) Fortenberry, R. C.; Huang, X.; Francisco, J. S.; Crawford, T. D.; Lee, T. J. Quartic force field predictions of the fundamental vibrational frequencies and spectroscopic constants of the cations $\mathrm{HOCO}+$ and DOCO+. J. Chem. Phys. 2012, 136, 234309.

(48) Obiosa-Maife, C.; Shaw, J. M. Toward Identification of Molecules in Ill-Defined Hydrocarbons Using Infrared, Raman, and Nuclear Magnetic Resonance (NMR) Spectroscopy. Energy Fuels 2011, 25, 460-471.

(49) Bauschlicher, C. W.; Langhoff, S. R. The calculation of accurate harmonic frequencies of large molecules: the polycyclic aromatic hydrocarbons, a case study. Spectrochim. Acta, Part A 1997, 53, 12251240.

(50) Saeki, M.; Akagi, H.; Fujii, M. Theoretical Study on the Structure and the Frequency of Isomers of the Naphthalene Dimer. J. Chem. Theory Comput. 2006, 2, 1176-1183.

(51) Malloci, G.; Joblin, C.; Mulas, G. On-line database of the spectral properties of polycyclic aromatic hydrocarbons. Chem. Phys. 2007, 332, 353-359.

(52) Michaelian, K. H.; Wen, Q.; Billinghurst, B. E.; Shaw, J. M.; Lastovka, V. Far- and mid-infrared photoacoustic spectra of tetracene, pentacene, perylene and pyrene. Vib. Spectrosc. 2012, 58, 50-56.

(53) Venuti, E.; et al. Phonons and structures of tetracene polymorphs at low temperature and high pressure. Phys. Rev. B 2004, 70, 104106.

(54) Campbell, R. B.; Robertson, J. M.; Trotter, J. The crystal structure of hexacene, and a revision of the crystallographic data for tetracene. Acta Crystallogr. 1962, 15, 289-290.

(55) Venuti, E.; Della Valle, R. G.; Brillante, A.; Masino, M.; Girlando, A. Probing Pentacene Polymorphs by Lattice Dynamics Calculations. J. Am. Chem. Soc. 2002, 124, 2128-2129.

(56) Brillante, A.; et al. Characterization of Phase Purity in Organic Semiconductors by Lattice-Phonon Confocal Raman Mapping: Application to Pentacene. Adv. Mater. 2005, 17, 2549-2553.

(57) Campbell, R. B.; Robertson, J. M.; Trotter, J. The crystal and molecular structure of pentacene. Acta Crystallogr. 1961, 14, 705-711.

(58) Holmes, D.; Kumaraswamy, S.; Matzger, A. J.; Vollhardt, K. P. C. On the Nature of Nonplanarity in the [N]Phenylenes. Chem.-Eur. J. 1999, 5, 3399-3412.
(59) Siegrist, T.; et al. Enhanced Physical Properties in a Pentacene Polymorph. Angew. Chem., Int. Ed. 2001, 40, 1732-1736.

(60) Mattheus, C. C.; et al. Polymorphism in pentacene. Acta Crystallogr., Sect. C 2001, 57, 939-941.

(61) Mattheus, C. C.; et al. Identification of polymorphs of pentacene. Synth. Met. 2003, 138, 475-481.

(62) Mattheus, C. C.; de Wijs, G. A.; de Groot, R. A.; Palstra, T. T. M. Modeling the Polymorphism of Pentacene. J. Am. Chem. Soc. 2003, 125, 6323-6330.

(63) Carbonniere, P.; Bégué, D.; Pouchan, C. DFT quartic force field of acetonitrile by using a generalized least-squares procedure. Chem. Phys. Lett. 2004, 393, 92-97.

(64) Becke, A. D. Density-functional thermochemistry. III. The role of exact exchange. J. Chem. Phys. 1993, 98, 5648-5652.

(65) Lee, C.; Yang, W.; Parr, R. G. Development of the Colle-Salvetti correlation-energy formula into a functional of the electron density. Phys. Rev. B 1988, 37, 785-789.

(66) Bauschlicher, C. W., Jr. A comparison of the accuracy of different functionals. Chem. Phys. Lett. 1995, 246, 40-44.

(67) Tirado-Rives, J.; Jorgensen, W. L. Performance of B3LYP Density Functional Methods for a Large Set of Organic Molecules. J. Chem. Theory Comput. 2008, 4, 297-306.

(68) Stephens, P. J.; Devlin, F. J.; Chabalowski, C. F.; Frisch, M. J. Ab Initio Calculation of Vibrational Absorption and Circular Dichroism Spectra Using Density Functional Force Fields. J. Phys. Chem. 1994, 98, 11623-11627.

(69) Gohaud, N.; Begue, D.; Pouchan, C. Vibrational spectra of methyllithium and its aggregates: a new interpretation from ab initio anharmonic calculations. Chem. Phys. 2005, 310, 85-96.

(70) Kristyán, S.; Pulay, P. Can (semi)local density functional theory account for the London dispersion forces? Chem. Phys. Lett. 1994, 229, $175-180$.

(71) Hobza, P.; Šponer, J.; Reschel, T. Density functional theory and molecular clusters. J. Comput. Chem. 1995, 16, 1315-1325.

(72) Begue, D.; Elissalde, S.; Pere, E.; Iratcabal, P.; Pouchan, C. New Theoretical and Experimental Infrared Results on Formaldehyde in Solution. J. Phys. Chem. A 2006, 110, 7793-7800.

(73) Bégué, D.; Qiao, G. G.; Wentrup, C. Nitrile Imines: Matrix Isolation, IR Spectra, Structures, and Rearrangement to Carbodiimides. J. Am. Chem. Soc. 2012, 134, 5339-5350.

(74) Pople, J. A.; Krishnan, R.; Schlegel, H. B.; Binkley, J. S. Derivative studies in hartree-fock and møller-plesset theories. Int. J. Quantum Chem. 1979, 16, 225-241.

(75) Chałasiński, G.; Szczęśniak, M. M. State of the Art and Challenges of the $a b$ Initio Theory of Intermolecular Interactions. Chem. Rev. 2000, 100, 4227-4252.

(76) Johnson, E. R.; DiLabio, G. A. Structure and binding energies in van der Waals dimers: Comparison between density functional theory and correlated ab initio methods. Chem. Phys. Lett. 2006, 419, 333339.

(77) Eilmes, A. A theoretical study of small tetracene clusters: Stability and charge stabilization energies. Comput. Theor. Chem. 2012, 982, 25-29.

(78) Chai, J.-D.; Head-Gordon, M. Long-range corrected hybrid density functionals with damped atom-atom dispersion corrections. Phys. Chem. Chem. Phys. 2008, 10, 6615-6620.

(79) Chai, J.-D.; Head-Gordon, M. Systematic optimization of longrange corrected hybrid density functionals. J. Chem. Phys. 2008, 128, 084106-1-084106-15.

(80) Salzner, U.; Aydin, A. Improved Prediction of Properties of $\pi$ Conjugated Oligomers with Range-Separated Hybrid Density Functionals. J. Chem. Theory Comput. 2011, 7, 2568-2583.

(81) Krishnan, R.; Binkley, J. S.; Seeger, R.; Pople, J. A. Selfconsistent molecular orbital methods. XX. A basis set for correlated wave functions. J. Chem. Phys. 1980, 72, 650.

(82) Frisch, M. J.; Pople, J. A.; Binkley, J. S. Self-consistent molecular orbital methods 25. Supplementary functions for Gaussian basis sets. J. Chem. Phys. 1984, 80, 3265. 
(83) Frisch, M.; et al. Gaussian 09, revision A.0; Gaussian, Inc.: Wallingford, CT, 2009.

(84) Bauschlicher, C. W., Jr.; Partridge, H. The sensitivity of B3LYP atomization energies to the basis set and a comparison of basis set requirements for CCSD (T) and B3LYP. Chem. Phys. Lett. 1995, 240, $533-540$.

(85) Bégué, D.; Gohaud, N.; Pouchan, C.; Cassam-Chenaï, P.; Liévin, J. A comparison of two methods for selecting vibrational configuration interaction spaces on a heptatomic system: Ethylene oxide. J. Chem. Phys. 2007, 127, 164115-1-164115-10.

(86) Bowman, J. M.; Carrington, T.; Meyer, H.-D. Variational quantum approaches for computing vibrational energies of polyatomic molecules. Mol. Phys. 2008, 106, 2145-2182.

(87) Bégué, D.; Baraille, I.; Garrain, P. A.; Dargelos, A.; Tassaing, T. Calculation of IR frequencies and intensities in electrical and mechanical anharmonicity approximations: Application to small water clusters. J. Chem. Phys. 2010, 133, 034102.

(88) Gohaud, N.; Begue, D.; Darrigan, C.; Pouchan, C. New parallel software ( $\mathrm{P}$ Anhar) for anharmonic vibrational calculations: Application to (CH3Li)2. J. Comput. Chem. 2005, 26, 743-754.

(89) Baraille, I.; Larrieu, C.; Dargelos, A.; Chaillet, M. Calculation of non-fundamental IR frequencies and intensities at the anharmonic level. I. The overtone, combination and difference bands of diazomethane, H2CN2. Chem. Phys. 2001, 273, 91-101.

(90) Cataldo, F.; Angelini, G.; Aníbal García-Hernández, D.; Manchado, A. Far infrared (terahertz) spectroscopy of a series of polycyclic aromatic hydrocarbons and application to structure interpretation of asphaltenes and related compounds. Spectrochim. Acta, Part A 2013, 111, 68-79.

(91) Malloci, G.; Mulas, G.; Cappellini, G.; Joblin, C. Timedependent density functional study of the electronic spectra of oligoacenes in the charge states $-1,0,+1$, and +2 . Chem. Phys. 2007, 340, 43-58.

(92) Brillante, A.; et al. Raman phonon spectra of pentacene polymorphs. Chem. Phys. Lett. 2002, 357, 32-36.

(93) Brock, C. P.; Dunitz, J. D. Temperature dependence of thermal motion in crystalline naphthalene. Acta Crystallogr., Sect. B 1982, 38, $2218-2228$

(94) Facelli, J. C.; Grant, D. M. Determination of molecular symmetry in crystalline naphthalene using solid-state NMR. Nature 1993, 365, 325-327.

(95) Brock, C. P.; Dunitz, J. D. Temperature dependence of thermal motion in crystalline anthracene. Acta Crystallogr., Sect. B 1990, 46, 795-806.

(96) Hineno, M.; Yoshinaga, H. Far-i.r. spectra of napthalene, anthracene, biphenyl, diphenyl ether, diphenylamine, diphenyl sulfoxide, and benzophenone at liquid helium temperature. Spectrochim. Acta, Part A 1975, 31, 617-620.

(97) Gonzalez, C.; Lim, E. C. A Quantum Chemistry Study of the van der Waals Dimers of Benzene, Naphthalene, and Anthracene: Crossed (D2d) and Parallel-Displaced (C2h) Dimers of Very Similar Energies in the Linear Polyacenes. J. Phys. Chem. A 2000, 104, 29532957.

(98) Tsuzuki, S.; Honda, K.; Uchimaru, T.; Mikami, M. High-level ab initio computations of structures and interaction energies of naphthalene dimers: Origin of attraction and its directionality. $J$. Chem. Phys. 2004, 120, 647-659.

(99) Walsh, T. . An ab initio study of the low energy structures of the naphthalene dimer. Chem. Phys. Lett. 2002, 363, 45-51.

(100) Rubeš, M.; Bludský, O.; Nachtigall, P. Investigation of the Benzene-Naphthalene and Naphthalene-Naphthalene Potential Energy Surfaces: DFT/CCSD(T) Correction Scheme. ChemPhysChem 2008, 9, 1702-1708.

(101) Simon, S.; Duran, M.; Dannenberg, J. J. How does basis set superposition error change the potential surfaces for hydrogen-bonded dimers? J. Chem. Phys. 1996, 105, 11024.

(102) Boys, S. F.; Bernardi, F. The calculation of small molecular interactions by the differences of separate total energies. Some procedures with reduced errors. Mol. Phys. 1970, 19, 553-566.
(103) Hohenstein, E. G.; Sherrill, C. D. Density fitting and Cholesky decomposition approximations in symmetry-adapted perturbation theory: Implementation and application to probe the nature of $\pi-\pi$ interactions in linear acenes. J. Chem. Phys. 2010, 132, 184111-1184111-10.

(104) Krainov, E. P. The Vibrational Spectra of Aromatic Compounds. Xix. Calculation and Interpretation of the Vibrational Spectra of Naphthalene and Some Deuterionaphthalenes. Opt. Spectrosc. 1964, 16, 415.

(105) Lutz, P. B.; Bayse, C. A. Orbital-based insights into paralleldisplaced and twisted conformations in $\pi-\pi$ interactions. Phys. Chem. Chem. Phys. 2013, 15, 9397-9406.

(106) Grimme, S. Do Special Noncovalent $\pi-\pi$ Stacking Interactions Really Exist? Angew. Chem., Int. Ed. 2008, 47, 3430-3434.

(107) Billinghurst, B.; et al. Photoacoustic spectroscopy using coherent synchrotron radiation: application to $\alpha$-lactose monohydrate. Opt. Lett. 2010, 35, 3090. 